\title{
Estimating the Division Rate of the Self-Similar Growth-Fragmentation Equation
}

\author{
Thibault Bourgeron* ${ }^{*}$ Marie Doumic ${ }^{\dagger}$ Miguel Escobedo Martínez ${ }^{\ddagger}$
}

August 26, 2013

\begin{abstract}
We consider the growth-fragmentation equation and we address the problem of finding the division rate from the stable size distribution of the population, which is easily measured, but non-smooth. We propose a method based on the Mellin transform for growth-fragmentation equations with self-similar kernels. We build a sequence of functions which converges to the density of the population in division, simultaneously in several weighted $\mathbb{L}^{2}$ spaces, as the measurement error goes to 0 . This improves previous results for self-similar kernels and allows us to understand the partial results for general fragmentation kernels. Numerical simulations confirm the theoretical results. Moreover, our numerical method is tested on real biological data, arising from a bacteria growth and fission experiment.
\end{abstract}

Keywords: Growth-fragmentation equations, Self-Similar Kernel, Regularization, Mellin Transform.

2010 Mathematics Subject Classification : 35Q92, 35R06, 45Q05, 65R10.

*Université Paris 6, laboratoire Jacques-Louis Lions, 4 place Jussieu, boîte courrier 187, 75252 Paris Cedex 05, France; email: bourgeron@ann.jussieu.fr.

${ }^{\dagger}$ INRIA Rocquencourt, équipe-projet BANG, domaine de Voluceau, BP 105, 78153 Rocquencourt, France; email: marie.doumic@inria.fr.

†Universidad del País Vasco, Facultad de Ciencias y Tecnología, Departamento de Matemáticas, Barrio Sarriena s/n 48940 Lejona (Vizcaya), Spain; email: miguel.escobedo@ehu.es. 


\section{Introduction}

A major issue for the coming years is to demonstrate the suitability of mathematical models to represent biological phenomena in a fully quantitative way. In this respect, inverse problem methods have already proved to be efficient. As a contribution to this field, and following previous studies [25, 14, 12, 11], this article proposes an improved method to calibrate a growth model of major importance: the growth-fragmentation equation. We also apply our method to experimental data of bacterial growth, as a proof of concept for its accuracy.

To describe the growth and division of particles over time, one of the key equation in the field of structured population dynamics is the so-called growth-fragmentation or cell division equation. This equation was first introduced at the end of the sixties to model cells dividing by fission [4], but it is also used to model protein polymerization [6], neuron networks $[22,23]$ and the TCP/IP window size protocol for the internet [1]. For all these application fields, the common point is that the "particle" under concern (which can be cells, polymers, dusts, windows, etc.) are well-characterized by their "size", i.e. a 1-dimensional quantity which grows over time, and which is distributed among the offspring of the particle when it divides.

The population is described by its concentration of particles of size $x$ at time $t$, denoted by $n(t, x)$. The equation for $n$ is obtained either by a mass balance, in the same spirit as for fluid dynamics [19, 3], or by considering the Kolmogorov equation for the underlying jumping process $[11,8]$ :

$$
\left\{\begin{array}{l}
\frac{\partial}{\partial t} n(t, x)+\frac{\partial}{\partial x}(g(x) n(t, x))=-B(x) n(t, x)+k \int_{x}^{\infty} \kappa(y, x) B(y) n(t, y) \mathrm{d} y, \quad x \geqslant 0, \\
n(0, x)=n^{0}(x), \quad g(0) n(t, 0)=0 .
\end{array}\right.
$$

Particles of size $x$ grow with a growth rate $g(x)$ and divide with a division rate $B(x)$. When a division occurs for a particle of size $y$, it splits into an average of $k>1$ smaller particles, giving rise to a particle of size $x$ with a probability rate $\kappa(x, y)$.

Given an initial data $n^{0} \in \mathbb{L}^{1}$, the existence of a unique solution $n$ in $C\left([0,+\infty) ; \mathbb{L}^{1}\right)$ to Equation (1) follows from classical analysis of transport equations, while additional integrability and $\mathbb{L}^{\infty}$ bounds may follow from the entropy structure of such models [20].

As concerns the asymptotic behaviour, under fairly general balance assumptions on the parameters $g, B$ and $\kappa$, it was proved that the population grows exponentially over time but tends to a steady profile, i.e. there exists a unique $\lambda>0$ and a unique function $N \in \mathbb{L}^{1}([0,+\infty))$ solution of the following eigenvalue problem:

$$
\left\{\begin{array}{l}
\frac{\partial}{\partial x}(g(x) N(x))+(B(x)+\lambda) N(x)-k \int_{0}^{+\infty} \kappa(x, y) B(y) N(y) \mathrm{d} y=0 \quad x \geqslant 0, \\
g(0) N(0)=0, \quad N(x>0)>0, \quad \lambda>0, \quad \int_{0}^{\infty} N(x) \mathrm{d} x=1,
\end{array}\right.
$$

and for a certain weighted norm, linked to the adjoint eigenvector, we have:

$$
e^{-\lambda t} n(t, x) \rightarrow N(x)
$$

see $[24,20,10]$ for more details, and recently $[21,22,23,18,5,2]$ for proof of an exponential speed of convergence. Remarkably, this trend to equilibrium is not only a mathematical result but is also supported by biological evidence [26], which may explain the very fast desynchronization of the cells, for instance [7].

For the problem that we are dealing with here, i.e. the calibration of the parameters of the equation, it is thus valid to consider that we start with a noisy measure of the steady 
behaviour, i.e. the exponential rate $\lambda$, also called the fitness of the population, and the steady profile $N(x)$. The way we can use a measure $\lambda_{\varepsilon}$ on the fitness $\lambda$ to determine the time-scale of the equation, for instance through a constant $c$ multiplying the growth rate $g(x)$, was explained in $[13,12]$; for the sake of simplicity, and in order to concentrate on the novel aspects of our study, we do not detail this aspect further, and here we consider that $\lambda>0$ as well as $g(x)$ are fully known. We model the measurement error in a deterministic way, assuming that we are given an observation $N^{\varepsilon}$ such that:

$$
\left\|N-N^{\varepsilon}\right\|_{\mathbb{H}^{s}([0,+\infty))} \leqslant \varepsilon
$$

where \|\|$_{\mathbb{H}^{s}([0,+\infty))}$ stands for the norm of a certain Sobolev Hilbert space $\mathbb{H}^{s}([0,+\infty))$.

As in the previous studies, we suppose that $g(x), k$ and $\kappa(y, x)$ are already known, or guessed at - such measures may be carried out directly in many cases [26] - and we concentrate on estimating the division rate $B(x)$.

We notice that $B$ only appears multiplied by $N$ in Equation (2), so that it cannot be accurately estimated from this equation where $N$ vanishes (near 0 and near $+\infty$ ). Hence, we denote $H=B N$ and in this article we focus on estimating $H$ rather than $B$ (the interested reader may find in [12] a fully rigorous estimate of $B$, obtained after a truncated division by $N$, i.e. by defining $B_{\eta}^{\varepsilon}=\frac{H^{\varepsilon} \mathbf{1}_{N^{\varepsilon} \geqslant \eta}}{N^{\varepsilon} \mathbf{1}_{N^{\varepsilon} \geqslant \eta}}$ ). Equation (2) may be formulated in terms of $H$ :

$$
\frac{\partial}{\partial x}(g(x) N(x))+\lambda N(x)=-H(x)+k \int_{0}^{+\infty} \kappa(x, y) H(y) \mathrm{d} y, \quad x \geqslant 0 .
$$

We can now formulate precisely the inverse problem under study, which the previous simplifications make linear.

Inverse Problem (IP): Given a measure $N^{\varepsilon}$ of the solution $N$ of Equation (2), if $N^{\varepsilon}$ satisfies Estimate (3), how can we get an approximation $H^{\varepsilon}$ of $H$ solution of Equation (4), and estimate the approximation error $\left\|H-H^{\varepsilon}\right\|_{\mathbb{L}^{2}\left(x^{q} \mathrm{~d} x\right)}$ in terms of $\varepsilon$, for small enough values of $q$ and for large enough values of $q$ ?

In $[25,14]$, a theoretical and numerical solution was proposed for the specific case of equal mitosis ${ }^{\dagger}$, i.e. when $k=2$ and $\kappa(x, y)=\delta_{x=\frac{y}{2}}$, and estimates were obtained in $\mathbb{L}^{2}([0,+\infty))$. In practice, the numerical solution exhibited an oscillatory behaviour for large values of $x$. Then, a generalization was proposed in [15] for a general division kernel $\kappa$, but the estimation was proved in spaces $\mathbb{L}^{2}\left(x^{q} \mathrm{~d} x\right)$ with $q>3$, which leads in practice to a noise amplification around zero. These two problems lead us to look for an estimate in a space that would guarantee a smooth behaviour both around zero and infinity: we are looking for an error estimate valid in $\mathbb{L}^{2}\left(x^{q} \mathrm{~d} x\right)$ spaces, with both $q=0$ and $q>3$, in order to avoid noise amplification around both zero and infinity.

The aim of this article is to give a complete solution of the inverse problem for general self-similar fragmentation kernels. Self-similarity refers here to the cases where fragmen-

${ }^{*}$ In articles $[25,14,15]$, the norm was in $\mathbb{L}^{2}$, i.e. $s=0$. In article [12], the noise coming from density estimation could be heuristically compared to $\mathbb{H}^{-1 / 2}$, i.e. $s=-1 / 2$.

$\dagger$ This kernel was the most widely studied (and is relevant for Escherichia coli or TCP/IP for instance). 
tation only depends on the ratio between the parent size and the offspring size. Mathematically, this means that there exists a probability measure $\kappa_{0}$ such that:

$$
\kappa(x, y)=\frac{1}{y} \kappa_{0}\left(\frac{x}{y}\right), \quad \kappa_{0} \in \mathcal{P}([0,1]), \quad \int_{0}^{1} z \mathrm{~d} \kappa_{0}(z)=\frac{1}{k}
$$

Let us give a weak formulation of Equation (4) when $\kappa$ satisfies Assumption (5): for every $\varphi$ in $C_{c}^{\infty}((0,+\infty))$ :

$$
-\int_{0}^{+\infty} g N \varphi^{\prime} \mathrm{d} x+\lambda \int_{0}^{+\infty} N \varphi \mathrm{d} x+\int_{0}^{+\infty} H \varphi \mathrm{d} x=k \int_{0}^{+\infty} H(x) \int_{0}^{1} \varphi(y x) \mathrm{d} \kappa_{0}(y) \mathrm{d} x .
$$

For self-similar fragmentation kernels, using the Mellin transform (cf. Section 3) provides a suitable tool, to obtain coherent theoretical and numerical estimations, and explains the previous partial results of $[25,14,15]$.

In the first section, we give the main steps required for regularizing and solving the inverse problem (IP), and state the main result. The second and third sections are devoted to the Mellin transform: we recall some fundamental results, which we use as the key point for our solution. The fourth section then details the numerical implementation. Technical details of the proofs are given in the appendix.

\section{Estimation Protocol and Main Result}

Let us briefly explain the main steps of our method. Since it was first introduced in [25] and then developed in $[14,15,12]$, we only outline its main features and we let the interested reader refer to those articles. First, we introduce some notations.

Definition $1.1\left(\mathbb{L}_{q}^{p}, \mathbb{H}^{n}, \mathbb{W}^{n, p}, \mathbb{W}^{-s, p}\right)$. Let $p \geqslant 1, q \in[0,+\infty), n \in \mathbb{N}^{*}$.

1. For $f: \mathbb{R} \rightarrow \mathbb{R}$ we denote

$$
\|f\|_{\mathbb{L}_{q}^{p}}:=\left(\int_{0}^{+\infty}|f(x)|^{p}|x|^{q} \mathrm{~d} x\right)^{1 / p} .
$$

We define $\mathbb{L}_{q}^{p}$ the Banach space $\mathbb{L}^{p}\left((0,+\infty), x^{q} \mathrm{~d} x\right)$ equipped with the norm \|\|$_{\mathbb{L}_{q}^{p}}$.

2. We denote

$$
\mathbb{W}^{n, p}\left(\left(1+x^{q}\right) \mathrm{d} x\right):=\left\{f:[0,+\infty) \rightarrow \mathbb{R} \mid f, \ldots, f^{(n)} \in \mathbb{L}^{p}\left(\left(1+x^{q}\right) \mathrm{d} x\right)\right\} .
$$

When equipped with the norm

$$
\|f\|_{\mathbb{W}^{n, p}\left(\left(1+x^{q}\right) \mathrm{d} x\right)}:=\sum_{k=0}^{n}\left\|f^{(k)}\right\|_{\mathbb{L}^{p}\left(\left(1+x^{q}\right) \mathrm{d} x\right)},
$$

$\mathbb{W}^{n, p}\left(\left(1+x^{q}\right) \mathrm{d} x\right)$ is a Banach space.

3. We define similarly $\mathbb{W}^{n, p}\left(x^{q} \mathrm{~d} x\right)$. We also define: $\mathbb{H}^{n}\left(\left(1+x^{q}\right) \mathrm{d} x\right)=\mathbb{W}^{n, 2}\left(\left(1+x^{q}\right) \mathrm{d} x\right)$ and $\mathbb{H}^{n}\left(x^{q} \mathrm{~d} x\right)=\mathbb{W}^{n, 2}\left(x^{q} \mathrm{~d} x\right)$. 
4. We also define:

$$
\mathbb{W}^{-1, p}\left(\left(1+x^{q}\right) \mathrm{d} x\right):=\left\{f \in \mathcal{D}^{\prime}(0,+\infty): f=g+h^{\prime}, g, h \in \mathbb{L}^{p}\left(\left(1+x^{q}\right) \mathrm{d} x\right)\right\} .
$$

Equipped with the norm:

$$
\|f\|_{\mathbb{W}^{-1, p}\left(\left(1+x^{q}\right) \mathrm{d} x\right)}:=\inf _{f=g+h^{\prime}}\left(\|g\|_{\mathbb{L}^{p}\left(\left(1+x^{q}\right) \mathrm{d} x\right)}+\|h\|_{\mathbb{L}^{p}\left(\left(1+x^{q}\right) \mathrm{d} x\right)}\right),
$$

$\mathbb{W}^{-1, p}\left(\left(1+x^{q}\right) \mathrm{d} x\right)$ is a Banach space.

5. Lastly we define for $\theta$ in $[0,1]$ the Banach space $\mathbb{W}^{-\theta, p}\left(\left(1+x^{q}\right) \mathrm{d} x\right)$ by complex interpolation:

$$
\mathbb{W}^{-\theta, p}\left(\left(1+x^{q}\right) \mathrm{d} x\right):=\left[\mathbb{L}^{p}\left(\left(1+x^{q}\right) \mathrm{d} x\right), \mathbb{W}^{-1, p}\left(\left(1+x^{q}\right) \mathrm{d} x\right)\right]_{\theta} .
$$

Equipped with the standard norm for complex interpolation spaces (see [30] for instance $), \mathbb{W}^{-\theta, p}\left(\left(1+x^{q}\right) \mathrm{d} x\right)$ is a Banach space.

The following linear operators, $\mathcal{K}, \mathcal{L}$ and $D$ were introduced in [14].

$$
\begin{aligned}
\mathcal{K}(H) & :=\int_{0}^{+\infty} \kappa(\cdot, y) H(y) \mathrm{d} y \\
\mathcal{L} & :=\operatorname{Id}-k \mathcal{K} . \\
D(N) & :=-\partial_{x}(g N)-\lambda N .
\end{aligned}
$$

Equation (4) may be formulated in terms of $\mathcal{L}$ and $D$ :

$$
\mathcal{L}(H)=D(N) .
$$

To solve the problem (IP), we thus need two steps. The first step is the same as in previous studies $[25,14,15,12]$, and consists in defining a convenient approximation $D_{\alpha}\left(N^{\varepsilon}\right)$ of $D(N)$ from a noisy measure $N^{\varepsilon}$. The second step is inverting the operator $\mathcal{L}$ : find $u$ solution to

$$
\mathcal{L}(u)=f .
$$

This is fully developed in Section 3, and it is the key point of the present article.

\section{First step: regularization and estimation of $D(N)$}

The operator $N \mapsto D(N)$ defined by (9) is homogeneous to a derivative operator so that it has a de-regularizing effect, whereas the operator $\mathcal{L}$ is shown in Section 3 to be continuously invertible from $\mathbb{L}_{q}^{p}$ to $\mathbb{L}_{q}^{p}$ spaces, under quite general assumptions on $p, q$. Hence, starting from a data $N^{\varepsilon}$ in $\mathbb{H}^{-s}([0,+\infty))$ for $0 \leqslant s \leqslant 1$, for $g$ which has a sufficient regularity we have: $D\left(N^{\varepsilon}\right) \in \mathbb{H}^{-s-1}([0,+\infty))$. To obtain an error estimate for $H-H^{\varepsilon}$ in an $\mathbb{L}^{2}$ space, we need a regularization step before applying $\mathcal{L}^{-1}$. This regularization being from $\mathbb{H}^{-s-1}$ to $\mathbb{L}^{2}=\mathbb{H}^{0}$ defines a degree of ill-posedness $a=1+s[16,31,12]$.

Among the many regularization techniques - see e.g. $[25,17]$ - we choose here the filtering or kernel approximation, already used in $[14,15,12]$ for its simplicity and accuracy. To do so, we introduce a mollifier $\rho$ satisfying:

$\rho \in C_{c}^{2}(\mathbb{R}), \quad \operatorname{supp} \rho \subset[-1,1], \quad \int_{-1}^{1} \rho(x) \mathrm{d} x=1, \quad \int_{-1}^{1} x^{k} \rho(x) \mathrm{d} x=0$ for $k=1, \ldots, n-1 ;$ 
(the forth condition is empty for $n=1$ ). We define $\rho_{\alpha}(x)=\frac{1}{\alpha} \rho\left(\frac{x}{\alpha}\right)$ that provides us with the natural approximation $D_{\alpha}\left(N^{\varepsilon}\right)$ of $D(N)$ :

$$
D_{\alpha}\left(N^{\varepsilon}\right):=\rho_{\alpha} * D\left(N^{\varepsilon}\right),
$$

which belongs to any $\mathbb{L}_{q}^{2}$; all the useful estimates are recalled in Lemma A.1 in the Appendix. We have the following estimate.

Proposition 1. Let $\rho$ satisfy Assumption (12). For $1 \leqslant p<\infty, 0 \leqslant q<\infty$ and $n \geqslant 1$, we suppose that $g N \in \mathbb{W}^{n+1, p}\left(\left(1+x^{q}\right) \mathrm{d} x\right)$ and $N \in \mathbb{W}^{n, p}\left(\left(1+x^{q}\right) \mathrm{d} x\right)$. Let $N^{\varepsilon} \in \mathbb{W}^{-s, p}\left(\left(1+x^{q}\right) \mathrm{d} x\right)$ with $0 \leqslant s \leqslant 1$. Defining $D(N)$ by (9) and $D_{\alpha}\left(N^{\varepsilon}\right)$ by (13) we have:

$$
\begin{aligned}
\left\|D_{\alpha}\left(N^{\varepsilon}\right)-D(N)\right\|_{\mathbb{L}_{q}^{p}} \leqslant C\left[\alpha^{-(1+s)}\left\|g\left(N^{\varepsilon}-N\right)\right\|_{\mathbb{W}-s, p}\left(\left(1+x^{q}\right) \mathrm{d} x\right)\right. & \\
& \left.+\alpha^{-s}\left\|N^{\varepsilon}-N\right\|_{\mathbb{W}^{-s, p}\left(\left(1+x^{q}\right) \mathrm{d} x\right)}+\alpha^{n}\right]
\end{aligned}
$$

where the constant $C$ depends on $2^{\frac{q-1}{p}},\left\|\rho^{\prime}\right\|_{\mathbb{L}^{1}([-1,1])},\left\|\rho^{\prime \prime}\right\|_{\mathbb{L}^{1}([-1,1])},|\lambda|$ and $\|g N\|_{\mathbb{W}^{n+1, p}\left(\left(1+x^{q}\right) \mathrm{d} x\right)}$, $\|N\|_{\mathbb{W}^{n, p}\left(\left(1+x^{q}\right) \mathrm{d} x\right)}$. If the terms $\left\|N^{\varepsilon}-N\right\|_{\mathbb{W}^{-s, p}\left(\left(1+x^{q}\right) \mathrm{d} x\right)},\left\|g\left(N^{\varepsilon}-N\right)\right\|_{\mathbb{W}^{-s, p}\left(\left(1+x^{q}\right) \mathrm{d} x\right)}$ are in the order of $\varepsilon$, the previous estimate is optimal for $\alpha=\varepsilon^{1 /(n+1+s)}$, which leads to:

$$
\left\|D_{\alpha}\left(N^{\varepsilon}\right)-D(N)\right\|_{\mathbb{L}_{q}^{p}}=O\left(\varepsilon^{n /(n+1+s)}\right) .
$$

\section{Second and main step: inversion of $\mathcal{L}$}

We now define a continuous inverse $\mathcal{L}^{-1}$ for $\mathcal{L}$, and apply it to $D_{\alpha}\left(N^{\varepsilon}\right)$ to obtain an estimate $H_{\alpha}^{\varepsilon}$ of $H$. In the case of a general fragmentation kernel, the operator $\mathcal{L}: \mathbb{L}_{q}^{2} \rightarrow \mathbb{L}_{q}^{2}$ was shown to be continuously invertible for $q>3$ in the article [15]. This result is generalised to $\mathbb{L}_{q}^{p}$ spaces, for large enough values of $q$, in Section $\mathrm{E}$ of the appendix. However, even if such a result provides an error estimate for $H-H_{\alpha}^{\varepsilon}$ in $\mathbb{L}_{q}^{2}$ spaces with $q>3$, it reveals unsatisfactory for practical purposes: the numerical methods developed in [15] from such definition of an inverse proved to blow-up around zero. To avoid such a blow-up, we would need to define an inverse in $\mathbb{L}_{q}^{2}$ spaces with smaller weights $q$, like $q=0$.

This was done for the case of the equal mitosis kernel $\kappa(y, x)=\delta_{x=\frac{y}{2}}$ in [14]: the operator $\mathcal{L}: \mathbb{L}_{q}^{2} \rightarrow \mathbb{L}_{q}^{2}$ was shown to be invertible for $q>3$ and also for $q<3$, with two distinct definitions of the inverse - one holding true for any $q<3$, the other for any $q>3$.

We extend this result of [14] to any self-similar kernel, by using the Mellin transform. We give here only the main ingredients, and refer to Sections 2 and 3 for full details.

Definition 1.2. Let $\mu$ be a complex valued regular measure on $(0,+\infty)$. The Mellin transform of $\mu$ at $s \in \mathbb{C}$ is defined by:

$$
\mathcal{M} \mu(s)=\int_{0}^{+\infty} x^{s-1} \mathrm{~d} \mu(x)
$$

provided that this expression exists. The Mellin transform of a function $f$ at $s \in \mathbb{C}$ is simply obtained by taking $\mu=f(x) \mathrm{d} x$ in the previous formula. 
The Mellin transform is suitable for the problem of inverting the linear operator $\mathcal{L}$ for a self-similar kernel $\kappa(x, y)=\frac{1}{y} \kappa_{0}\left(\frac{x}{y}\right)$ because the term $\mathcal{K} H=\int_{0}^{+\infty} \kappa_{0}(\dot{y}) H(y) \frac{\mathrm{d} y}{y}$ is the multiplicative convolution of the function $H$ and the measure $\kappa_{0}$. As the Fourier transform replaces additive convolution by product, the Mellin transform (which can be viewed as the standard Fourier transform when applying a logarithmic change of variables) replaces multiplicative convolution by product.

Thanks to (8) and to the relation (6) we have $\mathcal{M K} u(s)=\mathcal{M} \kappa_{0}(s) \mathcal{M} u(s)$ and the following identity holds:

$$
\mathcal{M L} u(s)=\left(1-k \mathcal{M} \kappa_{0}(s)\right) \mathcal{M} u(s) .
$$

Formally the solution $u$ of Equation (11) may be given by

$$
\mathcal{M} u(s)=\frac{\mathcal{M} f(s)}{1-k \mathcal{M} \kappa_{0}(s)}
$$

It remains to define a continuous inverse of the Mellin transform and to apply it to the right-hand side; this can be done on a vertical line of the complex plane. To do so we need $1-k \mathcal{M} \kappa_{0}$ not to vanish on such a vertical line; this is expressed in Assumption 1 below.

Assumption 1. $\zeta \in \mathbb{R}$ is such that the holomorphic function $1-k \mathcal{M} \kappa_{0}$ is bounded from below on the vertical line $\zeta+i \mathbb{R}$ by a positive constant.

Note that by Assumption (5) on $\kappa_{0}, 1-k \mathcal{M} \kappa_{0}(1)=1-k<0$ and $1-k \mathcal{M} \kappa_{0}(2)=0$, so that Assumption 1 never holds true for $\zeta=2$.

We then have the following proposition.

Proposition 2. If $q \in \mathbb{R}$ is such that $\zeta=\frac{q+1}{2}$ satisfies Assumption 1, the linear operator $\mathcal{L}$ defined by (8) is continuously invertible from $\mathbb{L}_{q}^{2}$ to $\mathbb{L}_{q}^{2}$.

This proposition is included in Proposition 5 of Section 3.2, where an upper bound for the norm of $\mathcal{L}^{-1}$ is also provided. In the article [14] (Appendix A.1) it is shown that the expression of $\mathcal{L}_{q}^{-1}: \mathbb{L}_{q}^{2} \rightarrow \mathbb{L}_{q}^{2}$ does not depend on $q>3$ or on $q<3$. We generalise this to self-similar kernels, $c f$. Propositions 4 and 6, respectively in $\mathbb{L}_{q}^{1}$ and in $\mathbb{L}_{q}^{2}$ spaces.

If $D(N) \in \mathbb{L}_{q}^{2}$, and if $B$ is such that $H=B N \in \mathbb{L}_{q}^{2}$, then $H=\mathcal{L}_{q}^{-1}(D(N))$ and we can define

$$
H_{q, \alpha}^{\varepsilon}:=\mathcal{L}_{q}^{-1}\left(D_{\alpha}\left(N^{\varepsilon}\right)\right)
$$

Proposition 2 together with Proposition 1 immediately give us an estimate for $\left\|H_{q, \alpha}^{\varepsilon}-H\right\|_{\mathbb{L}_{q}^{2}}$. However, as detailed in Propositions 4 and 6 of Section 3 below, such an inverse will not be the same in any space $\mathbb{L}_{q}^{2}$, due to the fact that the function $1-k \mathcal{M} \kappa_{0}$ has a zero at least in $s=2$ (which corresponds to the space $\mathbb{L}_{3}^{2}$ ). To obtain an estimate that would be true in several $\mathbb{L}_{q}^{2}$ spaces, for instance for both $q_{1}=0$ and $q_{2}>3$, we define for a given constant $a>0$ :

$$
H_{\alpha}^{\varepsilon}:=H_{q_{1}, \alpha}^{\varepsilon} \mathbf{1}_{[0, a]}+H_{q_{2}, \alpha}^{\varepsilon} \mathbf{1}_{[a,+\infty)} .
$$

To obtain an estimate for $H_{\alpha}^{\varepsilon}-H$, the last ingredient lies in the following interpolation lemma. 
Lemma 1.1. Let $a>0$, and $0 \leqslant q_{1} \leqslant q_{2}$. Let $f \in \mathbb{L}_{q_{1}}^{p}$ and $g \in \mathbb{L}_{q_{2}}^{p}$. Then the function $h=f \mathbf{1}_{[0, a]}+g \mathbf{1}_{[a,+\infty)}$ satisfies:

$$
\forall q \in\left[q_{1}, q_{2}\right], \quad\|h\|_{\mathbb{L}_{q}^{p}} \leqslant a^{\frac{q-q_{1}}{p}}\|f\|_{\mathbb{L}_{q_{1}}^{p}}+a^{-\frac{q_{2}-q}{p}}\|g\|_{\mathbb{L}_{q_{2}}^{p}}
$$

Proof. We first treat the case $a=1$. We have forall $x \geqslant 0$ :

$$
|h(x)|^{p} x^{q}=|f(x)|^{p} x^{q} \mathbf{1}_{[0,1]}+|g(x)|^{p} x^{q} \mathbf{1}_{(1,+\infty)} \leqslant|f(x)|^{p} x^{q_{1}} \mathbf{1}_{[0,1]}+|g(x)|^{p} x^{q_{2}} \mathbf{1}_{(1,+\infty)} .
$$

Integrating this inequality leads to $\|h\|_{\mathbb{L}_{q}^{p}}^{p} \leqslant\|f\|_{\mathbb{L}_{q_{1}}^{p}}^{p}+\|g\|_{\mathbb{L}_{q_{2}}^{p}}^{p}$ and the result follows from the convexity inequality $\left(|x|^{p}+|y|^{p}\right)^{\frac{1}{p}} \leqslant|x|+|y|$. The general case $a>0$ is obtained by a change of variable. We set $z_{a}(x)=z(a x)$ so that $z_{a}(1)=z(a)$. We apply the above inequality to the functions $f_{a}, g_{a}, h_{a}$ and the result is a consequence of the fact $\left\|z_{a}\right\|_{\mathbb{L}_{q}^{p}}=a^{-\frac{q+1}{p}}\|z\|_{\mathbb{L}_{q}^{p}}$

We can now state the main result of this article, which is a direct consequence of Propositions 1 and 2 and Lemma 1.1.

Theorem 1.1. Let $\kappa_{0}$ be defined by (5), and $q_{1}, q_{2}$ real numbers such that $0 \leqslant q_{1}<3<q_{2}$ and satisfying the assumptions of Proposition 2.

Let $(\lambda, N)$ with $N$ in $\mathbb{H}^{n}\left(\left(1+x^{q_{2}}\right) \mathrm{d} x\right)$ and $\lambda>0$ be the unique solution of Equation (2). We assume that: $g N \in \mathbb{H}^{n+1}\left(\left(1+x^{q_{2}}\right) \mathrm{d} x\right)$ and $B N \in \mathbb{L}^{2}\left(\left(1+x^{q_{2}}\right) \mathrm{d} x\right)$, for some $n \geqslant 1$.

Let $0 \leqslant s \leqslant 1$ and let $N^{\varepsilon}$ be a measure of $N$ such that $N^{\varepsilon} \in \mathbb{H}^{-s}\left(\left(1+x^{q}\right) \mathrm{d} x\right)$ for $q=q_{1}, q_{2}$ and with a measurement error given by:

$$
\left\|N^{\varepsilon}-N\right\|_{\mathbb{H}^{-s}\left(\left(1+x^{q}\right) \mathrm{d} x\right)} \leqslant \varepsilon, \quad\left\|g\left(N^{\varepsilon}-N\right)\right\|_{\mathbb{H}^{-s}\left(\left(1+x^{q}\right) \mathrm{d} x\right)} \leqslant \varepsilon .
$$

Let $a>0$ and let $\rho$ satisfy (12). We define $D_{\alpha}\left(N^{\varepsilon}\right)$ by (13), $H_{q, \alpha}^{\varepsilon}$ by (15) and $H_{\alpha}^{\varepsilon}$ by (16).

For every weight $q$ in $\left[q_{1}, q_{2}\right]$ and $0<\alpha \leqslant 1$, the following estimate holds:

$$
\left\|H_{\alpha}^{\varepsilon}-H\right\|_{\mathbb{L}_{q}^{2}} \leqslant C\left(\alpha^{-(1+s)} \varepsilon+\alpha^{n}\right)
$$

where the constant $C$ depends on $a, q,|\lambda|, q_{1}, q_{2},\left\|\rho^{\prime}\right\|_{\mathbb{L}^{1}([-1,1])},\left\|\rho^{\prime \prime}\right\|_{\mathbb{L}^{1}([-1,1])},\|N\|_{\mathbb{H}^{n}\left(\left(1+x^{q_{2}}\right) \mathrm{d} x\right)}$, $\|g N\|_{\mathbb{H}^{n+1}\left(\left(1+x^{q_{2}}\right) \mathrm{d} x\right)}$.

This induces an optimal error in the order of $\varepsilon^{n /(n+1+s)}$, obtained for a regularization parameter $\alpha$ in the order of $\varepsilon^{1 /(n+1+s)}$.

\section{Recall on the Mellin transform}

Notation 2.1. For a function $f: \zeta+i \mathbb{R} \rightarrow \mathbb{C}$ we denote

$$
\|f\|_{\mathbb{L}^{p}(\zeta+i \mathbb{R})}=\left(\int_{\mathbb{R}}|f(\zeta+i t)|^{p} \mathrm{~d} t\right)^{1 / p} .
$$

The construction of the Mellin transform on $i \mathbb{R}$ can be made in the general context of the Fourier transform on locally compact abelian groups (we refer the reader to Chapter 1 of [27]). Here we consider the multiplicative abelian group $G=(0,+\infty)$ (with unit 1 ), equipped with the topology inherited from $\mathbb{R}$ and with the Haar measure $\frac{\mathrm{d} x}{x}$ (that is 
the unique measure on $G$, up to a positive multiplicative constant, which is translationinvariant). It is easy to show that the dual group $\Gamma$ of all the characters of $G$ with the Gelfand topology is isomorphic to $i \mathbb{R}$ with the topology inherited from $\mathbb{C}$ via the application $i \mathbb{R} \rightarrow \Gamma, \quad i t \mapsto\left(x \mapsto x^{-i t}\right)$. In this general context the $\mathbb{L}^{1}$ and $\mathbb{L}^{2}$ theories can be built, with the same results, as the Fourier transform on the topological group $\left(\mathbb{R}^{n},+, \mathrm{d} x\right)$. The extension of the Mellin transform to a vertical line $\zeta+i \mathbb{R}$ of the complex plane $\mathbb{C}$ is simply obtained by defining $\mathcal{M} f(\zeta+i t)=\mathcal{M} g(i t)$ with $g(x)=x^{\zeta} f(x)$.

In the following subsection we state some simple definitions and results concerning the Mellin transform which will be useful for the proofs of Section 3. If a proposition cannot be found in [27] or in [29], a brief proof is sketched out in Appendix B. We first introduce the Mellin transform of a measure and then the Mellin transform of a function of class $\mathbb{L}^{1}, \mathbb{L}^{2}$.

\subsection{Mellin transform of a measure}

We recalled above in Definition 1.2 the Mellin transform of a measure. For the particular case of measures supported in $[0,1]$, this definition exists in a half-plane, as expressed in the following lemma.

Lemma 2.1. Let $\mu$ be a non-negative measure supported in $[0,1]$. If $\mathcal{M} \mu(a)$ exists for some real number a then $\mathcal{M} \mu$ is continuous in the closed half-plane $\{s \in \mathbb{C} \mid \operatorname{Re} s \geqslant a\}$ and is holomorphic in the open half-plane $\{s \in \mathbb{C} \mid \operatorname{Re} s>a\}$.

Definition 2.2. Let $\mu$ be a non-negative measure supported in $[0,1]$. We define the abscissa of convergence of $\mu$ as: $\operatorname{Abs} \mu=\inf \{a \in \mathbb{R} \mid \mathcal{M} \mu(a)$ is finite $\} \in \mathbb{R} \cup\{ \pm \infty\}$.

If $\operatorname{Abs} \mu$ is finite or $-\infty$ then $\mathcal{M} \mu$ is holomorphic in the half-plane $\{s \in \mathbb{C} \mid \operatorname{Re} s>$ Abs $\mu$ \}. However $\mathcal{M} \mu$ can be holomorphic in other strips of the complex plane, $c f$. Example 1 .

\section{Example 1.}

\begin{tabular}{|c|c|c|c|}
\hline self-similar kernel $\boldsymbol{\kappa}_{\mathbf{0}}$ & $\boldsymbol{\mathcal { M } \boldsymbol { \kappa } _ { \mathbf { 0 } } ( \boldsymbol { s } )}$ & Abs $\boldsymbol{\kappa}_{\mathbf{0}}$ & domain \\
\hline $\mathbf{1}_{[0,1]} \mathrm{d} x$ & $s^{-1}$ & 0 & $\mathbb{C} \backslash\{0\}$ \\
\hline$\alpha x^{\alpha-1} \mathbf{1}_{(0,1)} \mathrm{d} x$ & $\frac{\alpha}{\alpha+s-1}$ & $1-\alpha$ & $\mathbb{C} \backslash\{1-\alpha\}$ \\
\hline$\delta_{\sigma}, \sigma \in(0,1)$ & $\sigma^{s-1}$ & $-\infty$ & $\mathbb{C}$ \\
\hline$\frac{\alpha}{2}\left(x^{\alpha-1}+(1-x)^{\alpha-1}\right) \mathbf{1}_{(0,1)} \mathrm{d} x$, & $\frac{\alpha}{2}\left(\frac{1}{\alpha+s-1}+\mathrm{B}(s, \alpha)\right)$ & $\max (1-\alpha, 0)$ & $\operatorname{Re} s>0$, \\
$\alpha>0$ & & & $s \neq 1-\alpha$ \\
\hline
\end{tabular}

In the last line $\mathrm{B}(x, y)=\int_{0}^{1} t^{x-1}(1-t)^{y-1} \mathrm{~d} t$ is the Euler Beta function which is defined for $\operatorname{Re} x, \operatorname{Re} y>0$ (and linked to the Euler Gamma function by: $\mathrm{B}(x, y)=\frac{\Gamma(x) \Gamma(y)}{\Gamma(x+y)}$ ). The self-similar kernel $\kappa_{0}=\rho_{1} \delta_{\sigma_{1}}+\rho_{2} \delta_{\sigma_{2}}$ is studied in Appendix D.

Lemma 2.2. Let $\mu$ be a non-negative measure supported in $[0,1]$. The function $(\mathrm{Abs} \mu,+\infty) \rightarrow$ $\mathbb{R}, t \mapsto \mathcal{M} \mu(t)$

1. is a non-increasing and log-convex function,

2. tends to 0 as $t$ tends to $+\infty$ if $\mu$ is not of the form $a \delta_{1}$ for $a \in(0,+\infty)$.

3. is strictly decreasing if $\operatorname{supp}(\mu)$ is not included in $\{0,1\}$, 


\subsection{Mellin transform of functions on $\mathbb{C}$}

For $f$ in $\mathbb{L}_{q}^{1}$, we define the Mellin transform of $f$ by Definition 1.2 applied to $\mu=f(x) \mathrm{d} x$, for $s \in q^{*}+i \mathbb{R}$ with $q^{*}=q+1$ :

$$
\mathcal{M} f(s)=\int_{0}^{+\infty} x^{s} f(x) \frac{\mathrm{d} x}{x} .
$$

Theorem (Riemann-Lebesgue). The Mellin transform is a linear continuous map of $\mathbb{L}_{q}^{1}$ into $C_{b}^{0}\left(q^{*}+i \mathbb{R}\right) \subset \mathbb{L}^{\infty}\left(q^{*}+i \mathbb{R}\right)$, where $q^{*}=q+1$, with norm 1 .

Theorem (Inversion theorem). If $f$ is in $\mathbb{L}_{q}^{1}$ and if $\|\mathcal{M} f\|_{\mathbb{L}^{1}\left(q^{*}+i \mathbb{R}\right)}$ is finite, where $q^{*}=$ $q+1$, then for a.e. $x>0$ we have:

$$
f=\mathcal{M}_{q^{*}}^{-1}(\mathcal{M} f)
$$

where

$$
\mathcal{M}_{q^{*}}^{-1} \varphi(s):=\frac{1}{2 \pi i} \int_{q^{*}-i \infty}^{q^{*}+i \infty} \varphi(s) x^{-s} \mathrm{~d} s:=\frac{1}{2 \pi} \int_{\mathbb{R}} \varphi\left(q^{*}+i t\right) x^{-q^{*}} x^{-i t} \mathrm{~d} t .
$$

Lemma 2.3. If $f$ is in $\mathbb{L}_{q}^{1}$ for every real number $q$ in $(a, b)$ then its Mellin transform is holomorphic in the strip $\{s \in \mathbb{C} \mid a+1<\operatorname{Re} s<b+1\}$.

Example 2. 1. The function $f(x)=e^{-x}$ is in $\mathbb{L}_{q}^{1}$ for all $q>-1$ so $\mathcal{M} f(s)=\Gamma(s)$ is holomorphic in the half-plane $\{s \in \mathbb{C} \mid \operatorname{Re} s>0\}$.

2. The function $f(x)=\frac{1}{e^{x}-1}$ is in $\mathbb{L}_{q}^{1}$ for all $q>0$ so $\mathcal{M} f(s)=\Gamma(s) \zeta(s)$, where $\zeta(s)=\sum_{n=1}^{\infty} n^{-s}$ is the Riemann zeta function, is holomorphic in the half-plane $\{s \in \mathbb{C} \mid \operatorname{Re} s>1\}$.

Lemma 2.4. If $f$ is in $\mathbb{L}_{q}^{1}$ for every $q$ in $(a, b)$ then $\mathcal{M} f(\zeta+i t)$ tends to 0 as $|t|$ tends to $+\infty$ uniformly with respect to $\zeta \in[a+1+\eta, b+1-\eta]$, for any $0<\eta<\frac{b-a}{2}$.

Proposition. For a function $f$ in $\mathbb{L}_{q-1}^{1} \cap \mathbb{L}_{2 q-1}^{2}$ we have:

$$
\|f\|_{\mathbb{L}_{2 q-1}^{2}}=(2 \pi)^{-1 / 2}\|\mathcal{M} f\|_{\mathbb{L}^{2}(q+i \mathbb{R})} .
$$

This identity may be directly derived from the Plancherel theorem for the Fourier transform of $g(y)=f\left(e^{y}\right) e^{q y}$.

Theorem (Plancherel transform). According to the previous formula the Mellin transform can be extended, in a unique manner, to an isometry (up to the multiplicative constant $\left.(2 \pi)^{-1 / 2}\right)$ of $\mathbb{L}_{q}^{2}$ onto $\mathbb{L}^{2}(\widetilde{q}+i \mathbb{R})$, where $\widetilde{q}=\frac{q+1}{2}$.

A consequence of the previous construction of the Mellin transform in $\mathbb{L}^{2}$ is that for a function $f \in \mathbb{L}_{q-1}^{1} \cap \mathbb{L}_{2 q-1}^{2}$ (resp. $\left.\mathbb{L}^{2}(q+i \mathbb{R}) \cap \mathbb{L}^{1}(q+i \mathbb{R})\right)$ the two definitions of the Mellin transform (resp. the inverse Mellin transform) coincide. 


\section{Solution of Equation $\mathcal{L}(u)=f$ for self-similar ker- nels}

We are now equipped to give a rigorous meaning to Formula (14), which leads to solutions $u$ of Equation (11).

Using Section $2, \mathcal{M} \kappa_{0}$ is (at least) defined in the half-plane $\{s \in \mathbb{C} \mid \operatorname{Re} s \geqslant 1\}$ and satifies $\left|\mathcal{M} \kappa_{0}\right| \leqslant \frac{1}{k}$ in the half-plane $\{s \in \mathbb{C} \mid \operatorname{Re} s \geqslant 2\}$. Furthermore if $\operatorname{supp}\left(\kappa_{0}\right)$ is not included in $\{0,1\}$ then $\left|\mathcal{M} \kappa_{0}\right|<\frac{1}{k}$ in the half-plane $\{s \in \mathbb{C} \mid \operatorname{Re} s>2\}$ : Assumption 1 is always satisfied for $\zeta>2$.

The following assumption is equivalent to: $\mathcal{M}^{-1}\left(\frac{\mathcal{M} f(s)}{1-k \mathcal{M} \kappa_{0}(s)}\right) \in \mathbb{L}_{q}^{1}$. This assumption is not necessary in the $\mathbb{L}_{q}^{2}$ case.

Assumption 2. $q \in \mathbb{R}$ and $f \in \mathbb{L}_{q}^{1}$ are such that $\mathcal{M} f \in \mathbb{L}^{1}\left(q^{*}+i \mathbb{R}\right)$ with $q^{*}=q+1$ and there exists a function $v \in \mathbb{L}_{q}^{1}$ such that $\mathcal{M} f(s)\left(1-k \mathcal{M} \kappa_{0}(s)\right)^{-1}=\mathcal{M} v(s)$ for a.e. $s \in q+1+i \mathbb{R}$.

To investigate the link between the inverse Mellin transforms defined in $\mathbb{L}_{q}^{p}$ for different values of $q$, we need the following assumption, for both the $\mathbb{L}_{q}^{1}$ and $\mathbb{L}_{q}^{2}$ cases.

Assumption 3. For a given bandwidth $(a, b)$ with $a<2 \leqslant b$, let $c \in(a, 2]$ such that the zeros of $1-k \mathcal{M} \kappa_{0}$ in $\{s \in \mathbb{C} \mid a \leqslant \operatorname{Re} s \leqslant b\}$ are all included in the strip $\{s \in \mathbb{C} \mid c \leqslant$ $\operatorname{Re} s \leqslant 2\}$ (such a real number c exists as $\mathcal{M} \kappa_{0}$ is holomorphic and non-constant, cf. (5)).

We assume that: there exists a set of horizontal segments

$$
L_{n}=\left\{s \in \mathbb{C} \mid \operatorname{Im} s=c_{n} \text { and } c \leqslant \operatorname{Re} s \leqslant 2\right\},
$$

where $c_{n}$ tends to $\pm \infty$ when $n$ tends to $\pm \infty$, such that $1-k \mathcal{M} \kappa_{0}$ is bounded from below by a positive constant on $\cup_{n \in \mathbb{Z}} L_{n}$.

\subsection{Holomorphic Mellin transform in $\mathbb{L}^{1}$}

All the proofs in this section can be found in Appendices B and C, and strongly rely on the results recalled in Section 2.

The following proposition gives an explicit formula for $\mathcal{L}^{-1}: \mathbb{L}_{q}^{1} \rightarrow \mathbb{L}_{q}^{1}$ under some additional integrability assumptions.

Proposition 3. Let $q>\operatorname{Abs} \kappa_{0}-1$ such that $\zeta=q^{*}=q+1$ satisfies Assumption 1 . For every $f$ in $\mathbb{L}_{q}^{1}$ satisfying Assumption 2, there exists a unique $u \in \mathbb{L}_{q}^{1}$ solution to Equation (11). It is explicitly given for a.e. $x>0$ by:

$$
u(x):=\frac{1}{2 \pi i} \int_{q^{*}-i \infty}^{q^{*}+i \infty} \frac{\mathcal{M} f(s)}{1-k \mathcal{M} \kappa_{0}(s)} x^{-s} \mathrm{~d} s .
$$

If $f$ is in $\mathbb{L}_{q}^{1}$ for different values of $q$, does Formula (17) define the same solution $u$, whatever the value of $q$ is? And if not how can we characterize their difference? Remember that in [14] (Proposition A1), for the case $\kappa_{0}=\delta_{1 / 2}$, there were exactly two distinct solutions, one holding for any $q<1$ and one for any $q>1$. The following proposition generalises this result. 


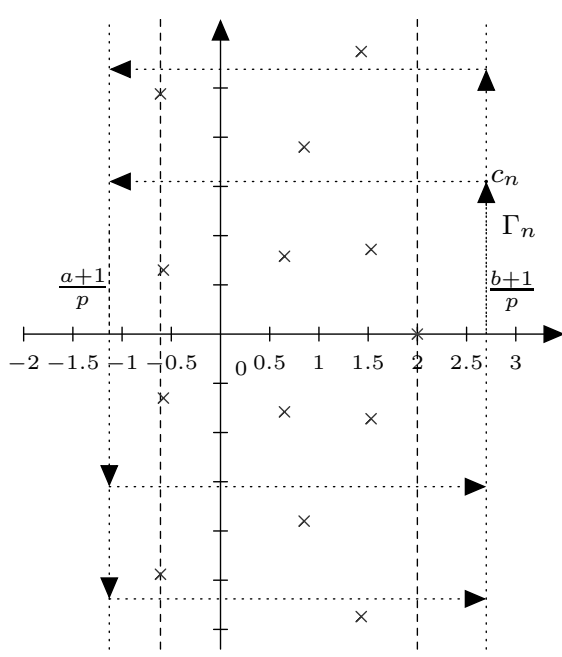

Figure 1: Proofs of Propositions 3, 5. The crosses are the zeros of $1-k \mathcal{M} \kappa_{0}$.

Proposition 4. Let $a, b$ be such that Abs $\kappa_{0}<a \leqslant 2<b$. We assume that the set $P$ of the zeros of $1-k \mathcal{M} \kappa_{0}$ satisfies Assumption 3 and that Assumption 1 is satisfied for any $\zeta=q^{*} \in(a, c) \cup(2, b)$. Let $f$ satisfy Assumption 2 for any $q \in(a-1, c-1) \cup(1, b-1)$.

For any $q \in(a-1, c-1) \cup(1, b-1)$, there exists a unique $u_{q} \in \mathbb{L}_{q}^{1}$ solution of Equation (11), given by the explicit formula (17). The solution $u_{q}=u_{l}(x)$ is independent of $q$ in $(a-1, c-1)$ (resp. $u_{q}=u_{r}(x)$ is independent of $q \in(1, b-1)$ ), and for almost every $x>0$ we have:

$$
u_{l}(x)-u_{r}(x)=\frac{1}{2 \pi} \sum_{p \in P} a_{p} \mathcal{M} f(p) x^{-p} \quad \text { with } a_{p}=\operatorname{Res}\left(\frac{1}{1-k \mathcal{M} \kappa_{0}(s)}, s=p\right) .
$$

Remark 3. Let $g=\left(1-k \mathcal{M} \kappa_{0}(s)\right)^{-1}$. From the relation $\overline{\mathcal{M} \kappa_{0}(s)}=\mathcal{M} \kappa_{0}(\bar{s})$ we deduce that the poles of $g$ are pairwise conjugated. Also, if $g$ can be represented as the Laurent series $g(s)=\sum_{n \in \mathbb{Z}} l_{n}(s-p)^{n}$ near $p$ then $g$ can be represented as the Laurent series $g(s)=\overline{g(\bar{s})}=\sum_{n \in \mathbb{Z}} \overline{l_{n}}(s-\bar{p})^{n}$ near $\bar{p}$; in particular $\overline{a_{p}}=a_{\bar{p}}$ with the notation of the theorem. Thus

$$
\sum_{p \in P} a_{p} \mathcal{M} f(p) x^{-p}=\sum_{p \in P \cap \mathbb{R}} a_{p} \mathcal{M} f(p) x^{-p}+2 \sum_{p \in P, \operatorname{Im} p>0} \operatorname{Re}\left(a_{p} \mathcal{M} f(p) x^{-p}\right) .
$$

Remark 4. Assume that for every $q^{*}$ in $(a, b)$ the relation $\left|1-k \mathcal{M} \kappa_{0}\left(q^{*}+i t\right)\right| \geqslant \delta_{q^{*}}>0$ is true for large values of $t$. Then, for every $q^{*}$ in $(a, b)$, either the continuous map $t \mapsto 1-k \mathcal{M} \kappa_{0}\left(q^{*}+i t\right)$ vanishes for some $t \in \mathbb{R}$ or there exists $\delta_{q^{*}}^{\prime}>0$ such that $\left|1-k \mathcal{M} \kappa_{0}\left(q^{*}+i t\right)\right| \geqslant \delta_{q^{*}}^{\prime}>0$. As $1-k \mathcal{M} \kappa_{0}$ is holomorphic in the strip $\{s \in \mathbb{C} \mid a<$ $\operatorname{Re} s<b\}$ the first case only occurs for a finite or countable set of values of $q^{*}$.

Remark 5. We have:

$$
u\left(e^{x}\right)=\frac{1}{2 \pi} e^{-q^{*} x} \int_{\mathbb{R}} g(t) e^{-i t x} \mathrm{~d} t \quad \text { where } \quad g(t)=\frac{\mathcal{M} f\left(q^{*}+i t\right)}{1-k \mathcal{M} \kappa_{0}\left(q^{*}+i t\right)} .
$$

The function $g$ is of class $C^{\infty}$, the method of the non-stationary phase, for the phase $\varphi(x)=-x$, shows that the term $\int_{\mathbb{R}} g(t) e^{-i t x} \mathrm{~d} t$ is in $O\left(x^{-n}\right)$ when $x$ tends to $+\infty$ for every 
integer $n \in \mathbb{N}$. Thus $u(x)$ is also in $O\left((\ln x)^{-n} x^{-q^{*}}\right)$ when $x$ tends to $+\infty$ for every integer $n \in \mathbb{N}$. The same argument with $u(\exp (-x))$ shows that $u(x)$ is in $O\left((-\ln x)^{-n} x^{-q^{*}}\right)$ when $x$ tends to 0 for every integer $n \in \mathbb{N}$.

Example 6. Consider $\kappa_{0}=\mathbf{1}_{[0,1]}, k=2$ and a function $f$ satisfying Assumption 2 for $q$ in $(0,2) \backslash\{1\}$. The functions $\mathcal{M} \kappa_{0}(s)=\frac{1}{s}$ and $\mathcal{M} f$ are holomorphic in the strip $\{s \in \mathbb{C} \mid$ $1<\operatorname{Re} s<3\}$. The function $1-2 \mathcal{M} \kappa_{0}$ only vanishes at $s=2$ and $\operatorname{Res}\left(\frac{s}{s-2}, s=2\right)=2$. For every $q^{*}$ in $(1,3), \mathcal{M} \kappa_{0}\left(q^{*}+i t\right)$ tends to $0 \neq \frac{1}{2}$ as $|t|$ tends to $+\infty$. Proposition 4 and Remark 4 show that, for almost every $x>0$ :

$$
u_{l}(x)-u_{r}(x)=\frac{1}{\pi} x^{-2} \int_{0}^{+\infty} t f(t) \mathrm{d} t .
$$

Example 7. Consider the general mitosis kernel $\kappa_{0}=\delta_{1 / k}$ for an integer $k \geqslant 2$ and a function $f$ in $\mathbb{L}_{q}^{1}$ for all $q$ in $(0,2)$ satisfying Assumption 2 for $q$ in $(0,2) \backslash\{1\}$. The functions $\mathcal{M} \kappa_{0}(s)=k^{1-s}$ and $\mathcal{M} f$ are holomorphic in the strip $\{s \in \mathbb{C} \mid 1<\operatorname{Re} s<3\}$. The set of the zeros of $1-k \mathcal{M} \kappa_{0}(s)$ is $2+\frac{2 \pi i}{\ln k} \mathbb{Z}$; it is easy to show that $\operatorname{Res}\left(\frac{1}{1-k^{2-s}}, 2+\frac{2 i \pi n}{\ln k}\right)=\frac{1}{\ln k}$ for every integer $n \in \mathbb{Z}$. For every $q$ in $(0,2) \backslash\{1\},\left|\mathcal{M} \kappa_{0}\left(q^{*}+i t\right)\right|=k^{-q} \neq \frac{1}{k}$. A simple computation leads to $\left|1-k \mathcal{M} \kappa_{0}(2+i y)\right|=2\left|\sin \left(y \frac{\ln k}{2}\right)\right|$; denoting $c_{n}=\frac{2 \pi n}{\ln k}$ for $n$ in $\mathbb{Z}$ we find that $1-k \mathcal{M} \kappa_{0}$ is bounded from below by 2 in $\cup_{n \in \mathbb{Z}}\left\{c_{n}\right\}$. Proposition 4 and Remark 4 show that, for almost every $x>0$ :

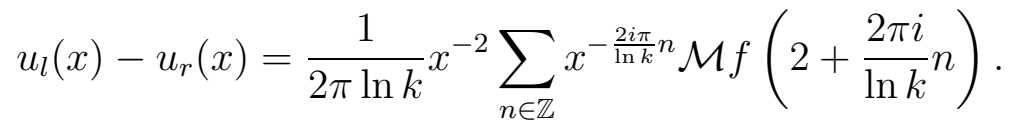

We refer the reader to Appendix $\mathrm{D}$ for a study of the zeros of $1-k \mathcal{M} \kappa_{0}$ if $\kappa_{0}=$ $\rho_{1} \delta_{\sigma_{1}}+\rho_{2} \delta_{\sigma_{2}}$.

\subsection{Isometric Mellin transform in $\mathbb{L}^{2}$}

We now turn to the $\mathbb{L}^{2}$ theory which is easier to handle in the context of inverse problems and in the direct continuation of the previous studies [25]. All the proofs of this section can be found in Appendix C.

In the $\mathbb{L}^{1}$ theory, we saw that the $\mathbb{L}_{q}^{1}$ norm for the function was linked to the $\mathbb{L}^{1}\left(q^{*}+i \mathbb{R}\right)$ space for its Mellin transform, with $q^{*}=q+1$. In the $\mathbb{L}_{q}^{2}$ theory, the $\mathbb{L}_{q}^{2}$ space of the function is linked to the space $\mathbb{L}^{2}(\widetilde{q}+i \mathbb{R})$ for its Mellin transform, with $\widetilde{q}=\frac{q+1}{2}$, as expressed by the Plancherel transform recalled in Section 2. The following proposition gives an explicit formula for $\left\|\mathcal{L}^{-1}\right\|_{\mathbb{L}_{q}^{2} \rightarrow \mathbb{L}_{q}^{2}}$.

Proposition 5. Let $q$ be a real number such that $\widetilde{q}=\frac{q+1}{2}>\operatorname{Abs} \kappa_{0}$ and Assumption 1 is satisfied for $\zeta=\widetilde{q}$. For every $f$ in $\mathbb{L}_{q}^{2}$ there exists a unique solution $u$ in $\mathbb{L}_{q}^{2}$ to Equation (11) and we have the estimate:

$$
\left\|\mathcal{L}^{-1}\right\|_{\mathbb{L}_{q}^{2} \rightarrow \mathbb{L}_{q}^{2}} \leqslant \sup _{t \in \mathbb{R}} \frac{1}{\left|1-k \mathcal{M} \kappa_{0}\left(\frac{q+1}{2}+i t\right)\right|} .
$$

Remark 8. A weak form of this proposition is the following. We have for all real number $t:\left|\mathcal{M} \kappa_{0}\left(\frac{q+1}{2}+i t\right)\right| \leqslant \mathcal{M} \kappa_{0}\left(\frac{q+1}{2}\right)$. Thus if: $\mathcal{M} \kappa_{0}\left(\frac{q+1}{2}\right)<\frac{1}{k},-$ which is true if $\operatorname{supp}\left(\kappa_{0}\right)$ is not included in $\{0,1\}$ and $q>3$ for instance - then:

$$
\sup _{t \in \mathbb{R}}\left|1-\mathcal{M} \kappa_{0}\left(\frac{q+1}{2}+i t\right)\right|^{-1} \leqslant\left|1-k \mathcal{M} \kappa_{0}\left(\frac{q+1}{2}\right)\right|^{-1} .
$$

This gives explicit expressions for $\left\|\mathcal{L}^{-1}\right\|_{\mathbb{L}_{q}^{2} \rightarrow \mathbb{L}_{q}^{2}}$ thanks to the table of Example 1 . 
As for the $\mathbb{L}^{1}$ theory, let us now turn to the case when $f$ is in $\mathbb{L}_{q}^{2}$ for several $q$. The following proposition corresponds to Proposition 4 in the $\mathbb{L}^{1}$-case.

Proposition 6. Let $a$ and $b$ be such that Abs $\kappa_{0}<a \leqslant 2<b$. We assume that the set $P$ of the zeros of $1-k \mathcal{M} \kappa_{0}$ satisfies Assumption 3 and that Assumption 1 is satisfied for any $\zeta=\widetilde{q}$ in $(a, c) \cup(2, b)$. Let $f \in \mathbb{L}_{q}^{2}$ for any $q$ in $(2 a-1,2 b-1)$.

For any $q$ in $(2 a-1,2 c-1) \cup(3,2 b-1)$, there exists a unique $u_{q}$ in $\mathbb{L}_{q}^{2}$ solution of Equation (11). For almost every $x>0, u_{q}(x)$ does not depend on $q$ in $(2 a-1,2 c-1)$ (resp. on $q$ in $(3,2 b-1))$.

Remark 9. Propositions 3, 4, 5 and 6 can be easily adapted to closed equations, such as the case of the conservative equation, where $\kappa_{0}$ is still defined by (5), but the system keeps only one fragment at each division, so that the number of fragments $k$ does not appear in front of the non-local term (2) and $\lambda=0$ :

$$
\left\{\begin{array}{l}
\frac{\partial}{\partial x}\left(g(x) N_{c}(x)\right)+B(x) N_{c}(x)-\int_{0}^{+\infty} \kappa(x, y) B(y) N_{c}(y) \mathrm{d} y=0 \quad x \geqslant 0 \\
g(0) N_{c}(0)=0, \quad N_{c}(x>0)>0, \quad \int_{0}^{\infty} N_{c}(x) \mathrm{d} x=1
\end{array}\right.
$$

In this case the linear operator $\mathcal{L}$ is replaced by $\mathcal{L}_{c}=\mathrm{Id}-\mathcal{K}$, so that $1-\mathcal{M} \kappa_{0}$ replaces $1-k \mathcal{M} \kappa_{0}$ in Assumptions 1, 2 and 3, and the "pivot vertical line" is $1+i \mathbb{R}$ instead of $2+i \mathbb{R}$. The exceptional spaces are $\mathbb{L}^{1}$ and $\mathbb{L}_{1}^{2}$ (resp. instead of $\mathbb{L}_{1}^{1}$ and $\mathbb{L}_{3}^{2}$ ). See also Remark 12.

\section{Numerical simulations}

\subsection{Protocol}

We assume that $k \in \mathbb{N}^{*}, g, \kappa$ are known. Given a measure $N^{\varepsilon}$ of $N$, we would like to compute approximations of the birth rate $B$. We define an approximation $D_{\alpha}\left(N^{\varepsilon}\right)$ of $D(N)$ by Formula (13).

Assuming that $N^{\varepsilon}$ is in $\mathbb{L}_{q}^{2}$ for $q$ in $\left[q_{1}, q_{2}\right], D_{\alpha}\left(N^{\varepsilon}\right)$ satisfies Assumptions 1 and 2 thanks to the regularity properties of the mollifier sequence $\left(\rho_{\alpha}\right)$. We can invert the operator $\mathcal{L}$ using the explicit formula (17) of Proposition 3 along the line $\frac{q+1}{2}+i \mathbb{R}$ for the two values $q=q_{1}, q_{2}$, and compute approximations $H_{q_{1}, \alpha}^{\varepsilon}=\mathcal{L}_{q_{1}}^{-1}\left(D_{\alpha}\left(N^{\varepsilon}\right)\right) \in \mathbb{L}_{q_{1}}^{2}$ and $H_{q_{2}, \alpha}^{\varepsilon}=$ $\mathcal{L}_{q_{2}}^{-1}\left(D_{\alpha}\left(N^{\varepsilon}\right)\right) \in \mathbb{L}_{q_{2}}^{2}$ of $H=B N$.

For a fixed $a>0$ we then compute $H_{\alpha, a}^{\varepsilon}$ by Formula (16).

Technical aspects. In order to compute integrals, we define the support of a function $f: \mathbb{R} \rightarrow \mathbb{C}$ as the set $f^{-1}(\{|f| \geqslant \eta\})$ where $\eta$ is a small positive constant. The integral of a compactly supported function is computed with a closed Newton-Cotes formula (namely the Boole-Villarceau's rule of order 5, cf. [9]). A computation with the Fast Fourier Transform is much faster (for $n$ points of integration only $O(n \ln n)$ operations are done instead of $O\left(n^{2}\right)$ with this method) but the results obtained are less accurate.

We denote $\mathcal{F} f(\xi)=\int_{0}^{+\infty} f(x) e^{-i x \xi} \mathrm{d} x$ the Fourier transform of a function $f$. Thanks to the relation $\mathcal{F} \rho_{\alpha}(\xi)=\mathcal{F} \rho(\alpha \xi)$, we compute the term $\rho_{\alpha} * D\left(N^{\varepsilon}\right)$ as:

$$
\left(\rho_{\alpha} *\left(g N^{\varepsilon}\right)\right)^{\prime}+\lambda \rho_{\alpha} * N^{\varepsilon}=\mathcal{F}^{-1}\left[\mathcal{F}(\alpha \xi)\left(-i \xi \mathcal{F}\left(g N^{\varepsilon}\right)(\xi)+\lambda \mathcal{F}\left(N^{\varepsilon}\right)(\xi)\right)\right]
$$


The Mellin transform, and its inverse, are also linked to the Fourier transforms thanks to the relations:

$$
\begin{aligned}
& \mathcal{M} f(c+i u)=\mathcal{F} g(u) \quad \text { where } g(z)=f(\exp (-z)) \exp (-c z), \\
& \mathcal{M}_{c}^{-1} f(x)=\frac{1}{2 \pi} x^{-c} \mathcal{F} h(\ln (x)) \quad \text { where } \quad h(z)=f(c+i z) .
\end{aligned}
$$

The terms appearing in Proposition 3 are computed in this way.

Before applying our method on biological data, see Section 4.3, we first tested it on simulations. We initially computed numerical approximations of the function $N$ using numerical schemes of the articles $[14,15]$, and then added a random noise to $N$. Let us denote $u^{\varepsilon}$ the uniform random variable on the interval $\frac{1}{2}[-1,1]$; we defined $N^{\varepsilon}=$ $\left(\max \left(N_{k}\left(1+u^{\varepsilon}\right), 0\right)\right)_{k \in\{1, \ldots, n\}}$ where $N=\left(N_{1}, \ldots, N_{n}\right)$ is the numerical approximation of the density $N$. We then applied the previously described technique to get a numerical approximation of $H$.

\subsection{Results on simulated data}

Parameters. For all the following simulations we selected the growth rate $g(x)=x^{1 / 2}$, and the birth rate to reconstruct $B(x)=x^{2}$. The self-similar kernels tested satisfy $k=2$ and are defined by $\kappa_{0}=\mathbf{1}_{[0,1]} \mathrm{d} x, \delta_{1 / 2}$ or $\frac{1}{\sqrt{2 \pi} \sigma} \exp \left(-\frac{1}{2 \sigma^{2}}\left(x-\frac{1}{2}\right)^{2}\right) \mathbf{1}_{[0,1]} \mathrm{d} x$ with $\sigma=0.1$. As proved theoretically in Section 3.1, the functions $1-2 \mathcal{M} \kappa_{0}$, for $\kappa_{0}=\mathbf{1}_{[0,1]} \mathrm{d} x, \delta_{1 / 2}$, only vanish on the vertical line $2+i \mathbb{R}$; this is also true numerically for the truncated Gaussian kernel $\frac{1}{\sqrt{2 \pi} \sigma} \exp \left(-\frac{1}{2 \sigma^{2}}\left(x-\frac{1}{2}\right)^{2}\right) \mathbf{1}_{[0,1]} \mathrm{d} x$. We took $a=2$.

We selected two mollifiers: $\rho_{1}=c \widetilde{\rho_{1}}$ with $\widetilde{\rho_{1}}(x)=\exp \left(\frac{1}{x(x-1)}\right) \mathbf{1}_{(0,1)}$ and $c=\left(\int_{0}^{1} \widetilde{\rho_{1}}(x) \mathrm{d} x\right)^{-1}$, and $\rho_{2}=c \widetilde{\rho_{2}}$ with $\widetilde{\rho_{2}}(x)=\exp \left(\frac{1}{x^{2}-1}\right) \mathbf{1}_{(-1,1)}$ and $c=\left(\int_{-1}^{1} \widetilde{\rho_{2}}(x) \mathrm{d} x\right)^{-1}$. The functions $\rho_{1}$ and $\rho_{2}$ are supported in $[-1,1]$ and are in $C^{\infty}(\mathbb{R})$. Moreover $\rho_{2}$ satifies $\int_{-1}^{1} x \rho_{2}(x) \mathrm{d} x=0$.

\section{Simulations and figures for each kernel $\kappa_{0}$.}

1. First no random noise is added to the numerically computed function $N$. Thus we perform the Mellin inversion along the two vertical lines $0.5+i \mathbb{R}$ and $3.5+i \mathbb{R}$. This gives two numerical approximations of $H$, respectively $H^{1}$ in $\mathbb{L}^{2}$ and $H^{2}$ in $\mathbb{L}_{6}^{2}$. We also define $H^{3}=H^{1} \mathbf{1}_{[0,2]}+H^{2} \mathbf{1}_{[2,+\infty)}$ which is in $\mathbb{L}_{q}^{2}$ for every $q$ in $[0,6]$. We can then approximate the birth rate $B$ by defining $B^{i}=H^{i} / \max (N, \vartheta)$ where $\vartheta$ is a threshold, see [12].

2. Then a random noise is added to the numerically computed function $N$ to get a noisy data $N^{\varepsilon}$ and we build numerical approximations $-H_{\alpha}^{1, \varepsilon} \in \mathbb{L}^{2}, H_{\alpha}^{2, \varepsilon} \in \mathbb{L}_{6}^{2}, H_{\alpha}^{3, \varepsilon} \in \mathbb{L}_{q}^{2}$ for every $q$ in $[0,6]$ - as previously. ing.

The mean convergence rates for $\left\|H_{\varepsilon}^{i}-H^{i}\right\|_{\mathbb{L}_{3}^{2}}, i=1,2,3$ and $\rho=\rho_{1}, \rho_{2}$ are the follow-

\begin{tabular}{|c|c|c|c|}
\hline$\rho_{\mathbf{1}}$ & $\left\|H_{\varepsilon}^{1}-H^{1}\right\|_{\mathbb{L}^{2}}$ & $\left\|H_{\varepsilon}^{2}-H^{2}\right\|_{\mathbb{L}_{6}^{2}}$ & $\left\|H_{\varepsilon}^{3}-H^{3}\right\|_{\mathbb{L}_{3}^{2}}$ \\
\hline $\mathbf{1}_{[0,1]} \mathrm{d} x$ & $12.26 \varepsilon^{0.73}$ & $110 \varepsilon^{0.62}$ & $24 \varepsilon^{0.68}$ \\
\hline$\delta_{1 / 2}$ & $5.76 \varepsilon^{0.61}$ & $90.15 \varepsilon^{0.68}$ & $23.21 \varepsilon^{0.68}$ \\
\hline$G$ & $15.23 \varepsilon^{0.73}$ & $91.77 \varepsilon^{0.68}$ & $22.53 \varepsilon^{0.68}$ \\
\hline
\end{tabular}



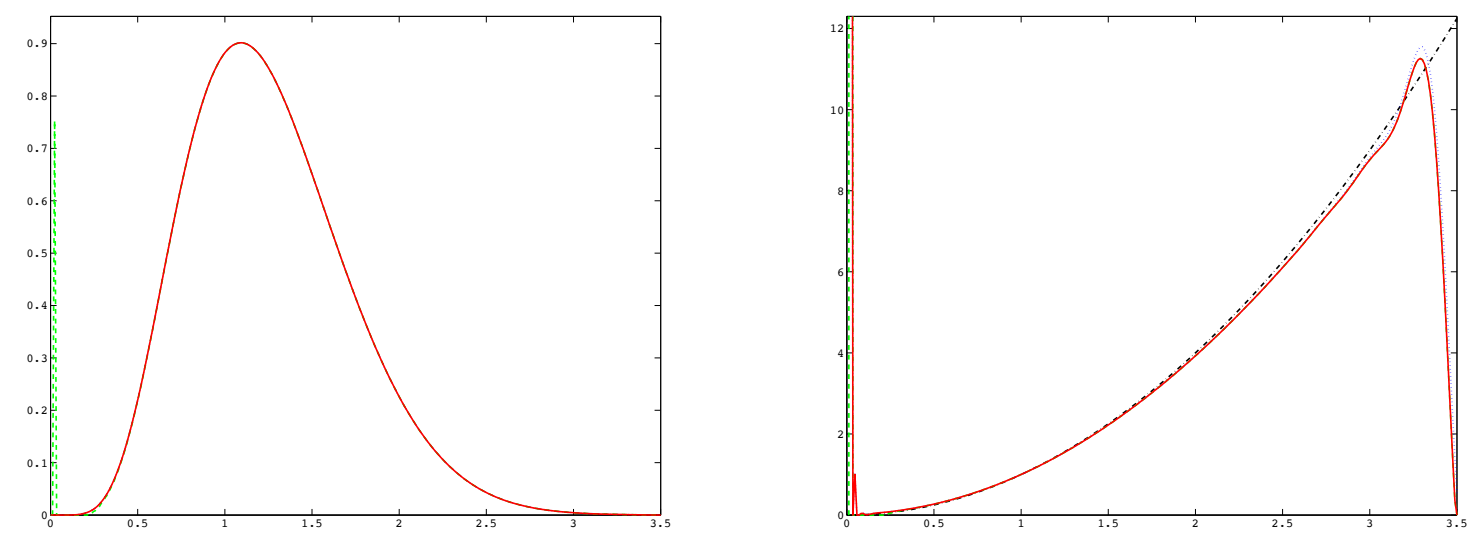

Figure 2: Reconstructions of $H$ and $B$ from exact numerical data $N$ for the truncated normal distribution and $\rho=\rho_{1}$. Dotted blue line: $H^{1}, B^{1}$; green dashed line: $H^{2}, B^{2}$; red line: $H^{3}, B^{3}$; dashed-dotted black line: $H=B N, B$.
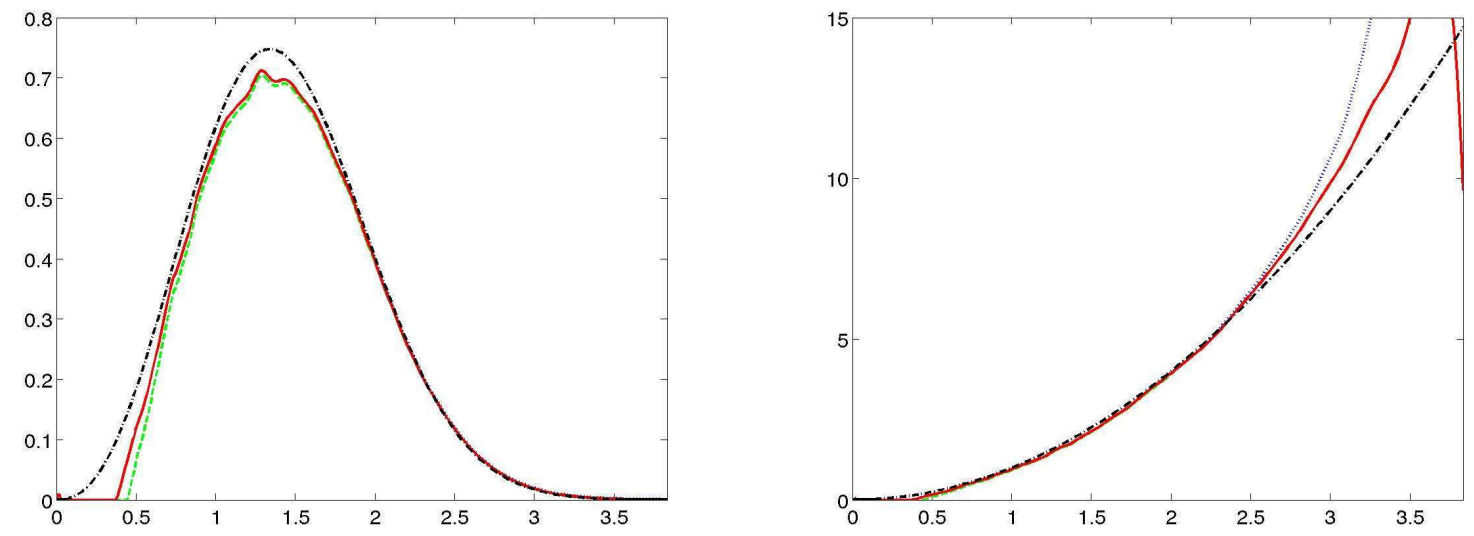

Figure 3: Reconstructions of $H$ and $B$ from noisy numerical data $N^{\varepsilon}$ for $\kappa_{0}=\mathbf{1}_{[0,1]} \mathrm{d} x,(\varepsilon, \alpha)=$ $(10 \%, 32 \%)$ and $\rho=\rho_{2}$.

\begin{tabular}{|c|c|c|c|}
\hline$\rho_{\mathbf{2}}$ & $\left\|H_{\varepsilon}^{1}-H^{1}\right\|_{\mathbb{L}^{2}}$ & $\left\|H_{\varepsilon}^{2}-H^{2}\right\|_{\mathbb{L}_{6}^{2}}$ & $\left\|H_{\varepsilon}^{3}-H^{3}\right\|_{\mathbb{L}_{3}^{2}}$ \\
\hline $\mathbf{1}_{[0,1]} \mathrm{d} x$ & $5.22 \varepsilon^{0.68}$ & $7.74 \varepsilon^{0.72}$ & $5.77 \varepsilon^{0.75}$ \\
\hline$\delta_{1 / 2}$ & $4.94 \varepsilon^{0.76}$ & $5.09 \varepsilon^{0.75}$ & $4.76 \varepsilon^{0.81}$ \\
\hline$G$ & $4.85 \varepsilon^{0.76}$ & $5.36 \varepsilon^{0.73}$ & $3.84 \varepsilon^{0.76}$ \\
\hline
\end{tabular}

All the illustrations are perfectly coherent with the theoretical results. The function $H^{2} \in \mathbb{L}_{6}^{2}$ is a bad approximation of $H=B N$ near 0 , whereas it is a better approximation of $H$ than $H^{1}$ near $+\infty$. The interpolated approximation $H^{3}$ is a good approximation of $H$ from both sides. The convergence rates of $\left(H_{i}^{\varepsilon}\right)$ obtained numerically are better than the expected theoretical results (for $\rho_{1}: O\left(\varepsilon^{0.6}\right)$ instead of $O\left(\varepsilon^{1 / 2}\right)$; for $\rho_{2}: O\left(\varepsilon^{0.7}\right)$ instead of $O\left(\varepsilon^{2 / 3}\right)$ ). Note that the constants numerically obtained for $\rho_{2}$ are smaller than the ones for $\rho_{1}$.

\subsection{Application to biological data}

We analysed a dataset obtained through a microscopic time-lapse imaging of Eschericia coli cells - data published in [28]. Since the cells divide into two almost equal daughter cells, this corresponds to $k=2$, and for the self-similar kernel $\kappa_{0}$, to an experimentally 

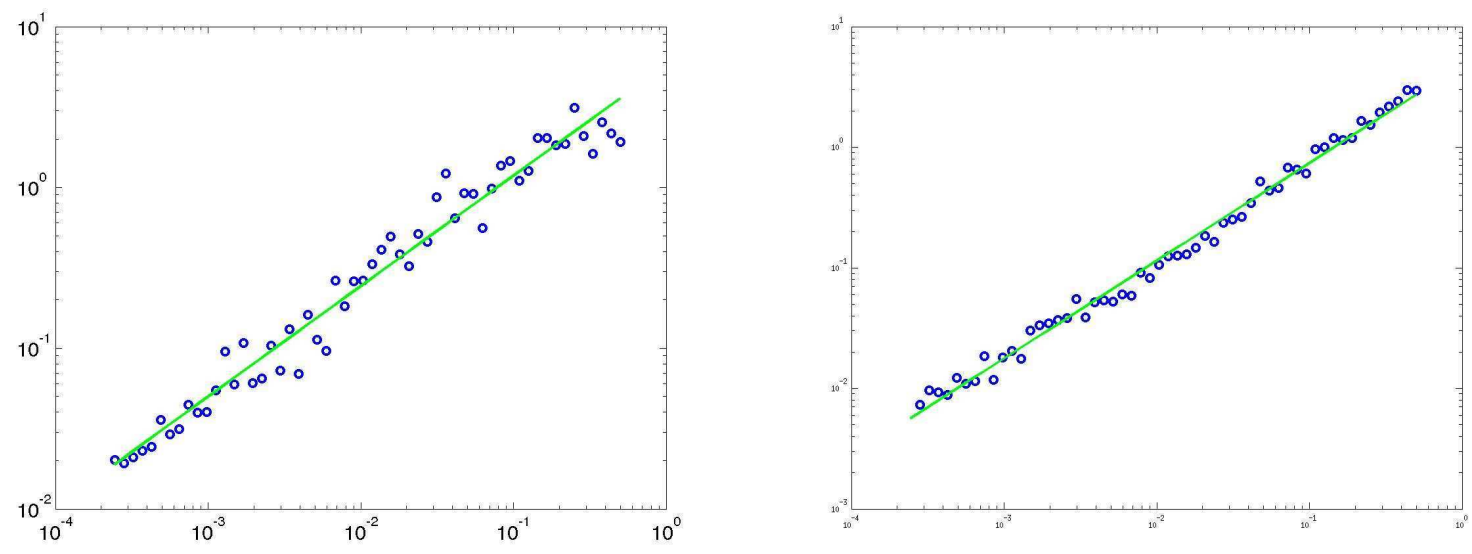

Figure 4: $\ln \left\|H_{\varepsilon}^{3}-H^{3}\right\|_{\mathbb{L}_{3}^{2}}$ as a function of $\ln \varepsilon$ for $\kappa_{0}=\delta_{1 / 2}$ and for $\rho=\rho_{1}$ (left), $\rho=\rho_{2}$ (right).
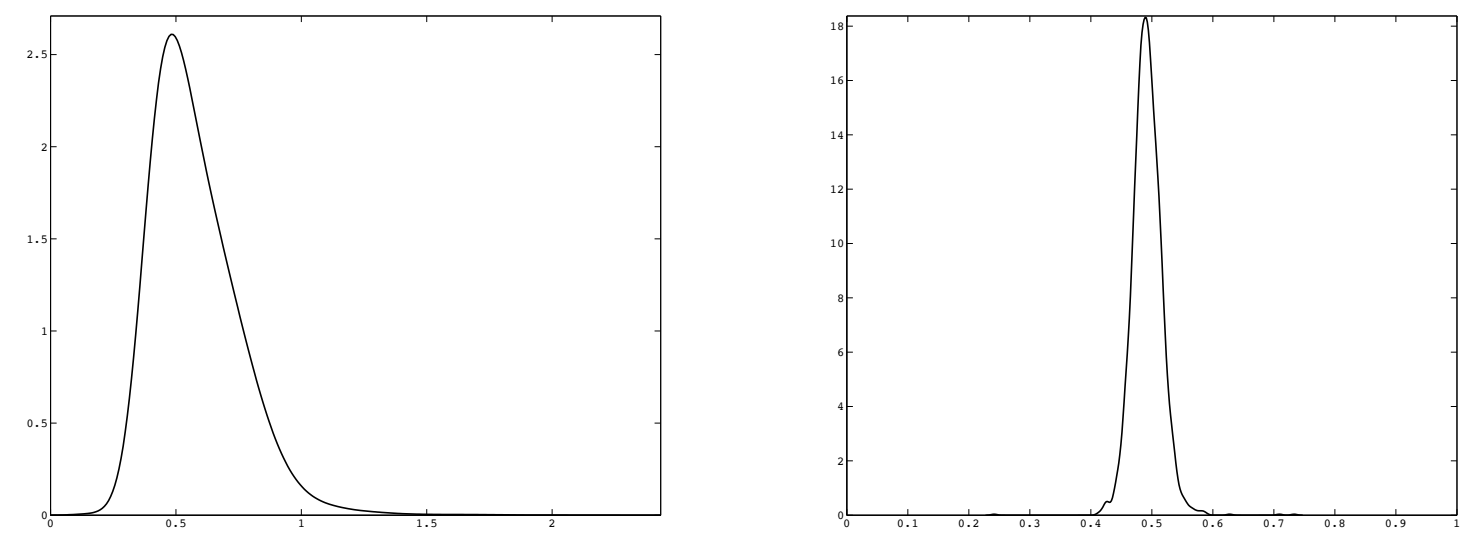

Figure 5: Reguralized density law $D_{\alpha}\left(N^{\varepsilon}\right)$ as a function of the sizes $x$ (left), with $\alpha=5.6$, and regularized experimentally measured fragmentation kernel $\kappa_{0}$ (right), with $\alpha=0.056$.

measured probability density $\kappa_{0}$ (see Figure 5, right). The growth rate of the cells is $g(x)=c x$, with a value of $c$ that can be experimentally measured - we took here $c=1$, which is always possible, up to a change in the timescale. In this case the parameter $\lambda$ is equal to $c$ so here $\lambda=1$. The experimental conditions are well-controlled and stable, so that the cells are in a steady state of growth. An image is taken every two minutes, and the length of each cell is measured through image analysis - since the cells are of cylindric shape with a roughly constant radius, we can identify the volume of a cell with its length, see $[11,26]$. In the dataset we analysed a sample of 30900 sizes, gathering all sizes at all times. As in [12], we model this sample as an $n$-sample of i.i.d. realizations of random variables of density $N(x)$, and we apply the mollifier directly to the dataset by defining

$$
D_{\alpha}\left(N^{\varepsilon}\right)(x):=\rho_{\alpha} * D\left(\frac{1}{n} \sum_{i=1}^{n} \delta_{x=x_{i}}\right),
$$

see Figure 5, left. The mollifier is the probability density function of the normal distribution $\mathcal{N}(0,1)$.

We also are able to measure the daughters' cell sizes just after division; we apply the same regularization technique to obtain the probability density $\kappa_{0}$. This fragmentation kernel is regularized with the optimal parameter for estimating normal densities. 

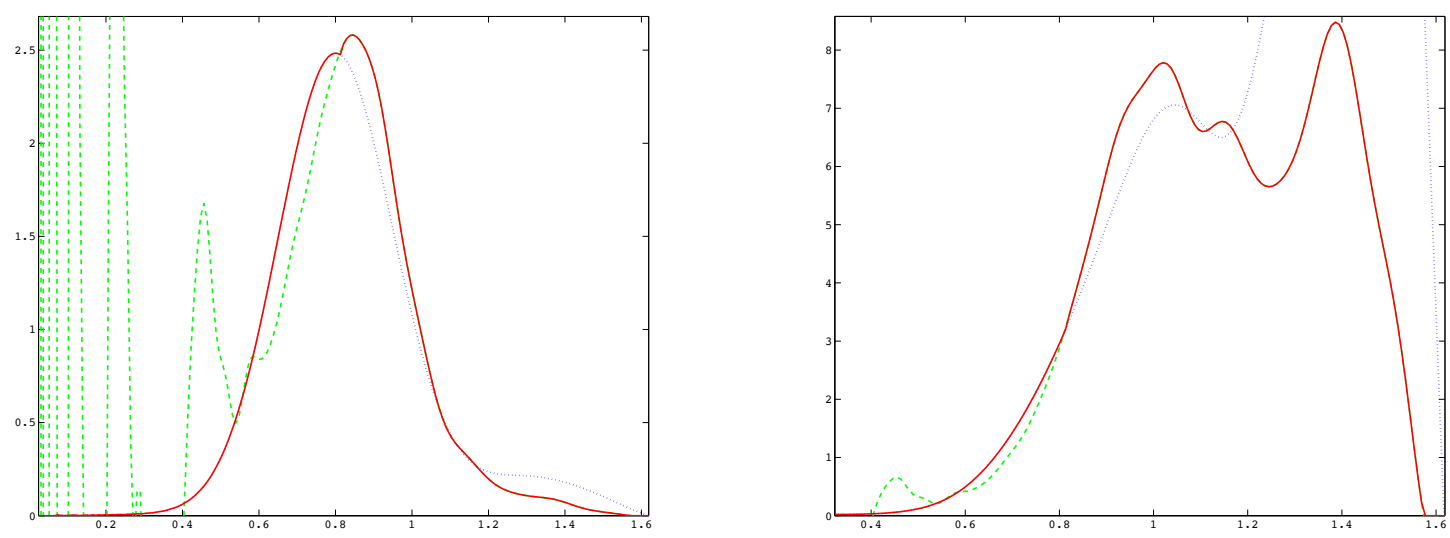

Figure $6: H^{i, \varepsilon}$ and $B^{i, \varepsilon}=\frac{H^{i, \varepsilon}}{N^{\varepsilon}}$ for $i=1,2,3$ as functions of the sizes $x$ for the optimal value of $\alpha$ and for $\kappa_{0}=\delta_{1 / 2}, a=0.81$.
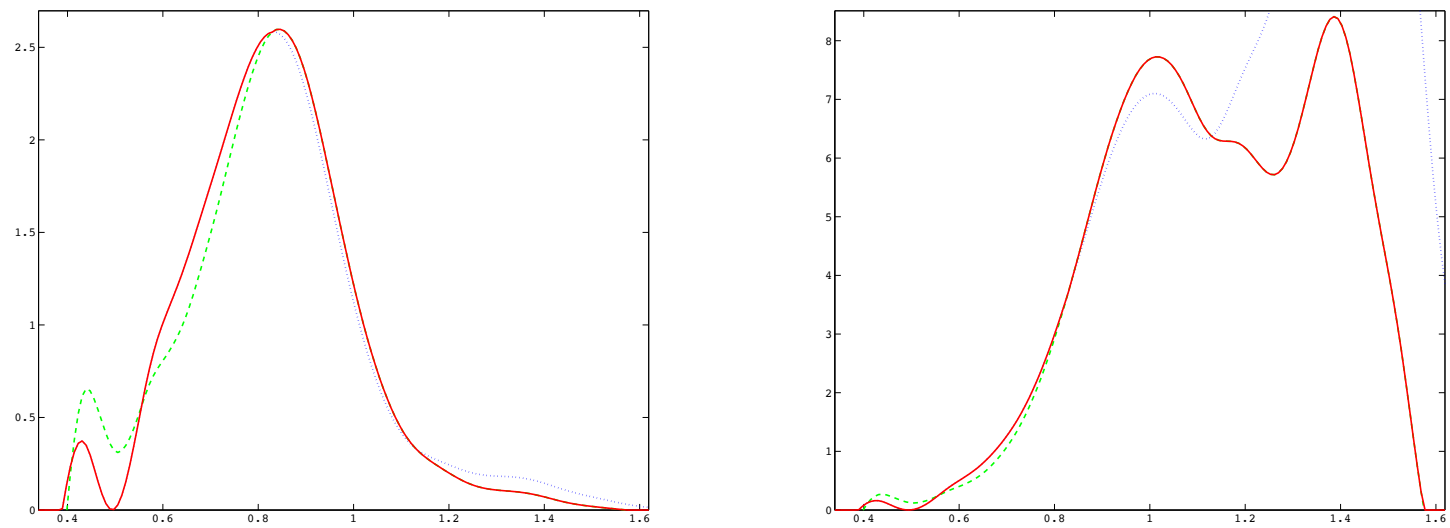

Figure 7: $H^{i, \varepsilon}$ and $B^{i, \varepsilon}=\frac{H^{i, \varepsilon}}{N^{\varepsilon}}$ for $i=1,2,3$ as functions of the sizes $x$ for the optimal value of $\alpha$ and for $\kappa_{0}$ which is experimentally measured, $a=0.83$.

To test our method, we first simulate the inverse problem with the regularized experimentally obtained steady state $D_{\alpha}\left(N^{\varepsilon}\right)$ and the fragmentation kernels $\kappa_{0}=\delta_{1 / 2}$ (see Figure 6) and $\kappa_{0}$ obtained experimentally (see Figure 7 ); we obtain the interpolated birth rate $B^{3, \varepsilon}$. Second, to measure the accuracy of this method, we simulate the direct problem with $B^{3, \varepsilon}$ and the two fragmentation kernels $\kappa_{0}$ (see Figure 8 ) ; we obtain the steady state $N\left(B^{3, \varepsilon}\right)$. To compare this steady state to $D_{\alpha}\left(N^{\varepsilon}\right)$ we define the discrepancy as:

$$
\frac{\left\|D_{\alpha}\left(N^{\varepsilon}\right)-N\left(B^{3, \varepsilon}\right)\right\|_{\mathbb{L}^{2}}}{\left\|D_{\alpha}\left(N^{\varepsilon}\right)\right\|_{\mathbb{L}^{2}}}
$$

We choose the paramater $\alpha$, for the regularization of $N^{\varepsilon}$, which minimizes the discrepancy. For both kernels the optimal value for $\alpha$ is the same: $\alpha=5.6$.

The discrepancies for both fragmentation kernels $\kappa_{0}$ are small, around $2 \%$. The discrepancy is slighltly smaller for the experimentally measured fragmentation kernel than for $\delta_{1 / 2}$. 

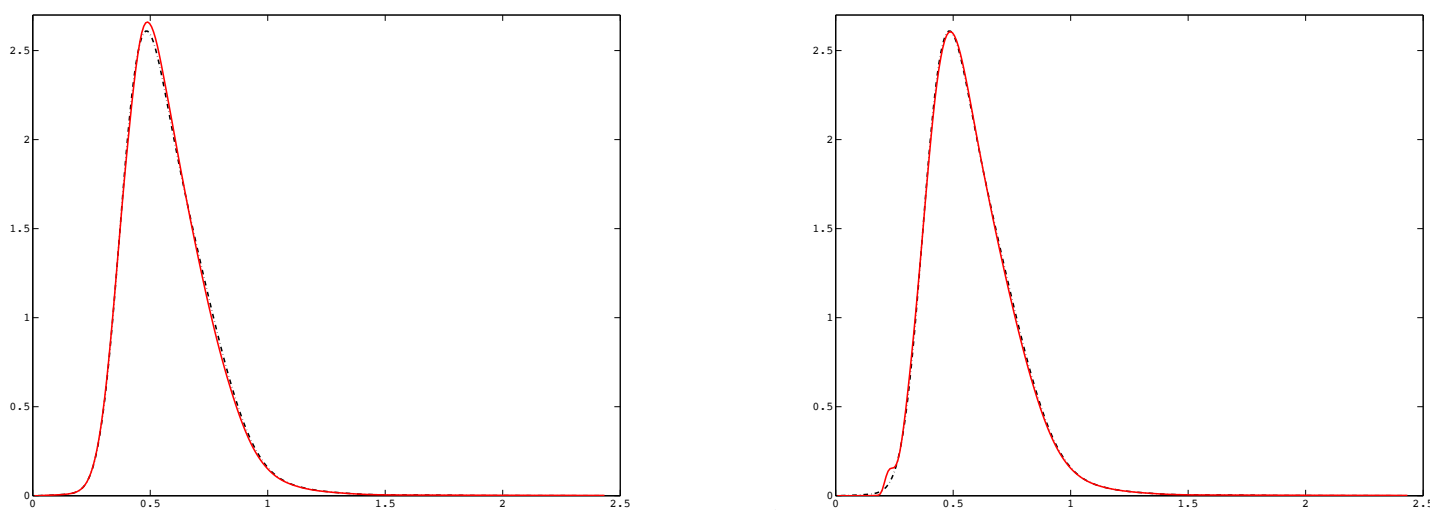

Figure 8: Density law $D_{\alpha}\left(N^{\varepsilon}\right)$ and steady states $N\left(B^{3, \varepsilon}\right)$ for the optimal value of $\alpha$, for $\kappa_{0}^{2}=\delta_{1 / 2}^{2.5}$ (left) and $\kappa_{0}$ experimentally measured (right). Discrepancies : $2.1 \%$ and $1.7 \%$.

\section{Conclusion}

We focussed on the problem of finding the birth rate $B$ of a size-structured population from measurements of the time-asymptotic profile $N^{\varepsilon}$, after [25], [14], [15]. For general fragmentation kernels we generalized the estimates, obtained in [15], to every $\mathbb{L}^{p}$ space for the birth rate $B$ and for less smooth data $N^{\varepsilon}$. Above all, for self-similar fragmentation kernels we provided a new method to find the birth rate $B$. The method is based on the Mellin transform, and is simple and efficient both theoretically and numerically. The method allowed us to understand the partial results for general fragmentation kernels and to improve them for self-semilar kernels. The results obtained numerically confirmed the theoretical estimates. The method made it possible to compute the birth rate $B$ from a fragmentation kernel which is experimentally measured. 


\section{A The filtering method: proof of Proposition 1}

The filtering method is classical both in a deterministic setting and in a statistical setting, where it is generally referred to as the kernel estimation method. We give here a quick proof for its application in our setting, for the sake of completeness. This method gives a control of $\left\|H_{\alpha}^{\varepsilon}-H\right\|_{\mathbb{L}_{q}^{p}}$ if the operator $\mathcal{L}: \mathbb{L}_{q}^{p} \rightarrow \mathbb{L}_{q}^{p}$ is invertible. We slightly generalise the statements of [15], where it was assumed that $N^{\varepsilon}$ is in $\mathbb{L}_{q}^{2}$, to a noisy measure $N^{\varepsilon} \in$ $\mathbb{W}^{-s, p}\left(\left(1+x^{q}\right) \mathrm{d} x\right)$ spaces, which allows us to compare our results directly with those obtained in a statistical setting (heuristically comparable to $s=-1 / 2$, see [12]).

We first recall some convolution estimates, which are the basis of kernel regularization methods. The following lemma is a kind of generalization of Lemma 2.1. of [15].

Lemma A.1. Let $p \geqslant 1, q \geqslant 0, \alpha \in[-1,1] \backslash\{0\}, \theta \in[0,1]$ real numbers and $\rho$ a function supported in $[-1,1]$. We define: $\rho_{\alpha}(x)=\frac{1}{\alpha} \rho\left(\frac{x}{\alpha}\right)$ for $|x| \leqslant \alpha$.

1. If the function $f$ is in $\mathbb{L}^{p}\left(\left(1+x^{q}\right) \mathrm{d} x\right)$ and if $\rho^{(n)}$ is in $\mathbb{L}^{1}([-1,1])$ for an integer $n \in \mathbb{N}$, we have:

$$
\left\|\left(\rho_{\alpha}\right)^{(n)} * f\right\|_{\mathbb{L}_{q}^{p}} \leqslant C|\alpha|^{-n}\|f\|_{\mathbb{L}^{p}\left(\left(1+x^{q}\right) \mathrm{d} x\right)},
$$

where $C=\max \left(1,2^{\frac{q-1}{p}}\right)\left\|\rho^{(n)}\right\|_{\mathbb{L}^{1}([-1,1])}$.

2. If the function $f$ is in $\mathbb{W}^{-\theta, p}\left(\left(1+x^{q}\right) \mathrm{d} x\right)$ and if $\rho, \rho^{\prime}$ are in $\mathbb{L}^{1}([-1,1])$, we have:

$$
\left\|\rho_{\alpha} * f\right\|_{\mathbb{L}_{q}^{p}} \leqslant C|\alpha|^{-\theta}\|f\|_{\mathbb{W}^{-\theta, p}\left(\left(1+x^{q}\right) \mathrm{d} x\right)},
$$

where $C=\max \left(1,2^{\frac{q-1}{p}}\right) \max \left(\|\rho\|_{\mathbb{L}^{1}([-1,1])},\left\|\rho^{\prime}\right\|_{\mathbb{L}^{1}([-1,1])}\right)$.

3. If the function $f$ is in $\mathbb{W}^{-\theta, p}\left(\left(1+x^{q}\right) \mathrm{d} x\right)$ and if $\rho^{\prime}, \rho^{\prime \prime}$ are in $\mathbb{L}^{1}([-1,1])$, we have:

$$
\left\|\left(\rho_{\alpha}\right)^{\prime} * f\right\|_{\mathbb{L}_{q}^{p}} \leqslant C|\alpha|^{-(1+\theta)}\|f\|_{\mathbb{W}-\theta, p\left(\left(1+x^{q}\right) \mathrm{d} x\right)},
$$

where $C=\max \left(1,2^{\frac{q-1}{p}}\right) \max \left(\left\|\rho^{\prime}\right\|_{\mathbb{L}^{1}([-1,1])},\left\|\rho^{\prime \prime}\right\|_{\mathbb{L}^{1}([-1,1])}\right)$.

4. If $f$ is in $\mathbb{W}^{1, p}\left(\left(1+x^{q}\right) \mathrm{d} x\right)$ and if $\rho$ is in $\mathbb{L}^{1}([-1,1])$ with $\|\rho\|_{\mathbb{L}^{1}([-1,1])}=1$ we have:

$$
\left\|f-\rho_{\alpha} * f\right\|_{\mathbb{L}_{q}^{p}} \leqslant C|\alpha|\|f\|_{\mathbb{W} 1, p\left(\left(1+x^{q}\right) \mathrm{d} x\right)},
$$

where $C=\max \left(1,2^{\frac{q-1}{p}}\right)$.

5. Let $n \in \mathbb{N}$ an integer. If $f$ is in $\mathbb{W}^{n+1, p}\left(\left(1+x^{q}\right) \mathrm{d} x\right)$ and if $\rho$ satisfies $\|\rho\|_{\mathbb{L}^{1}([-1,1])}=1$ and $\int_{-1}^{1} z^{k} \rho(z) \mathrm{d} z=0$ for $k=1, \ldots, n$ we have:

$$
\left\|f-\rho_{\alpha} * f\right\|_{\mathbb{L}_{q}^{p}} \leqslant C|\alpha|^{n+1}\|f\|_{\mathbb{W}^{n+1, p}\left(\left(1+x^{q}\right) \mathrm{d} x\right)},
$$

where $C=\max \left(1,2^{\frac{q-1}{p}}\right) /(n !)$. 
Proof of Lemma A.1. We use the standard inequalities, valid for all real numbers $x, y$ and $\alpha \geqslant 0$ :

$$
\begin{array}{lrl}
\alpha \geqslant 1 & |x|^{\alpha}+|y|^{\alpha} \leqslant(|x|+|y|)^{\alpha} \leqslant 2^{\alpha-1}\left(|x|^{\alpha}+|y|^{\alpha}\right) \\
\alpha \in[0,1] & 2^{\alpha-1}\left(|x|^{\alpha}+|y|^{\alpha}\right) & \leqslant(|x|+|y|)^{\alpha}
\end{array}
$$

1. We first prove this inequality in the case $n=0$. Writing $|\rho|=|\rho|^{\frac{1}{p^{\prime}}}|\rho|^{\frac{1}{p}}$ and by Hölder's inequality, we have:

$$
\left|\rho_{\alpha} * f(x)\right|=\left|\int_{-1}^{1} \rho(z) f(x-\alpha z) \mathrm{d} z\right| \leqslant\|\rho\|_{\mathbb{L}^{1}([-1,1])}^{\frac{1}{p^{\prime}}}\left(\int_{-1}^{1}|\rho(z)||f(x-\alpha z)|^{p} \mathrm{~d} z\right)^{\frac{1}{p}} .
$$

Then:

$$
\begin{aligned}
\left\|\rho_{\alpha} * f\right\|_{\mathbb{L}_{q}^{p}}^{p} & \leqslant\|\rho\|_{\mathbb{L}^{1}([-1,1])}^{\frac{p}{p^{\prime}}} \int_{-1}^{1} \int_{0}^{+\infty}|f(x-\alpha z)|^{p}|\rho(z)||x|^{q} \mathrm{~d} x \mathrm{~d} z \\
& =\|\rho\|_{\mathbb{L}^{1}([-1,1])}^{\frac{p}{p^{\prime}}} \int_{-1}^{1}|\rho(z)| \int_{0}^{+\infty}|f(x-\alpha z)|^{p}|x-\alpha z+\alpha z|^{q} \mathrm{~d} x \mathrm{~d} z \\
& \leqslant\|\rho\|_{\mathbb{L}^{1}([-1,1])}^{\frac{p}{p^{p}}} \int_{-1}^{1}|\rho(z)| \int_{0}^{+\infty}|f(x-\alpha z)|^{p} C\left(|\alpha|^{q}+|x-\alpha z|^{q}\right) \mathrm{d} x \mathrm{~d} z \\
& \leqslant C\|\rho\|_{\mathbb{L}^{1}([-1,1])}^{p}\left(\|f\|_{\mathbb{L}_{q}^{p}}^{p}+|\alpha|^{q}\|f\|_{\mathbb{L}^{p}}^{p}\right) \\
& \leqslant C\|\rho\|_{\mathbb{L}^{1}([-1,1])}^{p}\|f\|_{\mathbb{L}^{p}\left(\left(1+x^{q}\right) \mathrm{d} x\right)}^{p},
\end{aligned}
$$

where $C=\max \left(1,2^{q-1}\right)$. The general case $n \in \mathbb{N}$ is a consequence of the previous inequality using the fact that $\left(\rho_{\alpha}\right)^{(n)}=\alpha^{-n}\left(\rho^{(n)}\right)_{\alpha}$.

2. We first prove the inequality for $\theta=1$ and then use an interpolation argument to get it for $\theta$ in $[0,1]$. Let $f=g+h^{\prime}$ a function in $\mathbb{W}^{-1, p}\left(\left(1+x^{q}\right) \mathrm{d} x\right)$ with $f, g$ in $\mathbb{L}^{p}\left(\left(1+x^{q}\right) \mathrm{d} x\right)$. Writing $\left\|\rho_{\alpha} * f\right\|_{\mathbb{L}_{q}^{p}} \leqslant\left\|\rho_{\alpha} * g\right\|_{\mathbb{L}_{q}^{p}}+\left\|\left(\rho_{\alpha}\right)^{\prime} * h\right\|_{\mathbb{L}_{q}^{p}}$, applying the previous inequality for $n=0,1$ and taking the infimum over $g$, $h$ such that $f=g+h^{\prime}$ we get the result. Let $\theta \in[0,1]$ and let $T=\rho_{\alpha} *$. The first point of the lemma with $n=0$ implies that $T$ is bounded from $\mathbb{L}_{q}^{p}$ to $\mathbb{L}^{p}\left(\left(1+x^{q}\right)\right)$ with its norm less than $\max \left(1,2^{\frac{q-1}{p}}\right)\|\rho\|_{\mathbb{L}^{1}([-1,1])} \leqslant C$ where $C=\max \left(1,2^{\frac{q-1}{p}}\right) \max \left(\|\rho\|_{\mathbb{L}^{1}([-1,1])},\left\|\rho^{\prime}\right\|_{\mathbb{L}^{1}([-1,1])}\right)$. We have just proved that $T$ is bounded from $\mathbb{L}_{q}^{p}$ to $\mathbb{W}^{-1, p}\left(\left(1+x^{q}\right) \mathrm{d} x\right)$ with its norm less than $C \frac{1}{|\alpha|}$. Therefore by complex interpolation and by definition of $\mathbb{W}^{-\theta, p}\left(\left(1+x^{q}\right) \mathrm{d} x\right)$ we get the estimate.

3. This is the same proof as that of point 2 with $\rho^{\prime}$ instead of $\rho$.

4. As we have $f, f^{\prime} \in \mathbb{L}^{p}$ we can write: $f(x)-f(x-\alpha z)=\alpha z \int_{0}^{1} f^{\prime}(x-t \alpha z) \mathrm{d} t$, so, by Hölder's inequality or by Jensen's inequality: $|f(x)-f(x-\alpha z)|^{p} \leqslant|\alpha z|^{p} \int_{0}^{1}\left|f^{\prime}(x-t \alpha z)\right|^{p} \mathrm{~d} t$. As $\left\|\rho_{\alpha}\right\|_{\mathbb{L}^{1}([-1,1])}=1$ we have:

$$
\begin{aligned}
\left\|f-\rho_{\alpha} * f\right\|_{\mathbb{L}_{q}^{p}}^{p} & =\int_{0}^{+\infty}\left|\int_{-1}^{1}(f(x)-f(x-\alpha z)) \rho(z) \mathrm{d} z\right|^{p}|x|^{q} \mathrm{~d} x \\
& \leqslant \int_{0}^{+\infty} \int_{-1}^{1}|f(x)-f(x-\alpha z)|^{p}|\rho(z)| \mathrm{d} z|x|^{q} \mathrm{~d} x \\
& \leqslant \int_{-1}^{1} \int_{0}^{1} \int_{0}^{+\infty}\left|f^{\prime}(x-t \alpha z)\right|^{p}|\alpha|^{p}|\rho(z)||x|^{q} \mathrm{~d} x \mathrm{~d} t \mathrm{~d} z
\end{aligned}
$$


the rest of the proof is the same as that given in the first point of the lemma with $f^{\prime}$ instead of $f$.

5. As we have $f, \ldots, f^{n+1} \in \mathbb{L}^{p}$ we can write, for a.e. $x \in[0,+\infty)$ :

$$
f(x)-f(x-\alpha z)=\sum_{k=1}^{n} \frac{1}{k !} f^{(k)}(x-\alpha z)(\alpha z)^{k}+\frac{1}{n !}(\alpha z)^{n+1} \int_{0}^{1} t^{n} f^{(n+1)}(x-t \alpha z) \mathrm{d} t .
$$

Integrating this quantity multiplied by $\rho(z)$ we get:

$$
f(x)-\rho_{\alpha} * f(x)=\frac{\alpha^{n+1}}{n !} \int_{-1}^{1} z^{n+1} \rho(z) \int_{0}^{1} t^{n} f^{(n+1)}(x-t \alpha z) \mathrm{d} t \mathrm{~d} z .
$$

By the same manipulation as for the previous point we get:

$$
\left\|f-\rho_{\alpha} * f\right\|_{\mathbb{L}_{q}^{p}}^{p} \leqslant \frac{|\alpha|^{(n+1) p}}{(n !)^{p}} \int_{-1}^{1} \int_{0}^{1} \int_{0}^{+\infty}\left|f^{(n+1)}(x-t \alpha z)\right|^{p}|\rho(z)||x|^{q} \mathrm{~d} x \mathrm{~d} t \mathrm{~d} z,
$$

which leads to the conclusion as previously.

Proof of Proposition 1. Between $D(N)$ defined by (9) and $D_{\alpha}\left(N^{\varepsilon}\right)$ by (13), we define an intermediate $D_{\alpha}$ by

$$
D_{\alpha}=\rho_{\alpha} * D(N)
$$

We use the decomposition: $\left\|D_{\alpha}\left(N^{\varepsilon}\right)-D(N)\right\|_{\mathbb{L}_{q}^{p}} \leqslant\left\|D_{\alpha}\left(N^{\varepsilon}\right)-D_{\alpha}(N)\right\|_{\mathbb{L}_{q}^{p}}+\left\|D_{\alpha}(N)-D(N)\right\|_{\mathbb{L}_{q}^{p}}$.

1. For the term $\left\|D_{\alpha}\left(N^{\varepsilon}\right)-D_{\alpha}(N)\right\|_{\mathbb{L}_{q}^{p}}$ it remains to estimate the two terms: $\left\|\left(\rho_{\alpha} *\left(g N^{\varepsilon}-g N\right)\right)^{\prime}\right\|_{\mathbb{L}_{q}^{p}}$ and $|\lambda|\left\|\rho_{\alpha} *\left(N^{\varepsilon}-N\right)\right\|_{\mathbb{L}_{q}^{p}}$ which can be done using respectively the third point of the lemma for $\theta=s$ and the second point for $\theta=s$.

2. For the term $\left\|D_{\alpha}(N)-D(N)\right\|_{\mathbb{L}_{q}^{p}}$ we write:

$$
\begin{aligned}
\left\|\left(\rho_{\alpha} *(g N)\right)^{\prime}-\lambda \rho_{\alpha} * N-\left((g N)^{\prime}-\lambda N\right)\right\|_{\mathbb{L}_{q}^{p}} & \\
& \leqslant\left\|\rho_{\alpha} *(g N)^{\prime}-(g N)^{\prime}\right\|_{\mathbb{L}_{q}^{p}}+|\lambda|\left\|\rho_{\alpha} * N-N\right\|_{\mathbb{L}_{q}^{p}}
\end{aligned}
$$

and we use the fourth point or the fifth point of the lemma.

For $a, b>0, \alpha, \varepsilon>0$ we write: $\varepsilon \alpha^{-a}+\alpha^{b}=\alpha^{(b-a) / 2} \varepsilon^{1 / 2}\left(\varepsilon^{1 / 2} \alpha^{-(b+a) / 2}+\varepsilon^{-1 / 2} \alpha^{(b+a) / 2}\right)$; the second term is greater than 2 with equality iff $\alpha=\varepsilon^{1 /(b+a)}$. Thus we obtain the inequality:

$$
\varepsilon \alpha^{-a}+\alpha^{b} \geqslant 2 \varepsilon^{b /(b+a)}
$$

which is an equality iff $\alpha=\varepsilon^{1 /(b+a)}$. The last points of the proposition are consequences of this inequality with $(a, b)=(1+s, 1)$ and $(a, b)=(1+s, n+1)$. 


\section{B Proofs of the lemmas of Section 2}

Proof of Lemma 2.1. This is a consequence of Lebesgue's dominated convergence for holomorphic functions. For every real number $x>0: s \mapsto x^{s-1}$ is holomorphic in $\mathbb{C}$ and for every $s$ in $\mathbb{C}$ such that $\operatorname{Re} s \geqslant a:\left|x^{s-1}\right| \leqslant x^{a-1} \in \mathbb{L}^{1}([0,1], \mu)$.

Proof of Lemma 2.2. The proofs are based on Lebesgue's dominated convergence theorem and on Hölder's inequality.

1. If $a>b$, for all $x$ in $[0,1]: x^{a} \leqslant x^{b}$. If $k=\alpha k_{1}+(1-\alpha) k_{2}$ with $\alpha$ in $(0,1)$ the inequality $\mathcal{M} \kappa_{0}(k) \leqslant \mathcal{M} \kappa_{0}\left(k_{1}\right)^{\alpha} \mathcal{M} \kappa_{0}\left(k_{2}\right)^{1-\alpha}$ is a consequence of Hölder's inequality for $p=\frac{1}{\alpha} \in[1,+\infty]$.

2. For $x$ in $[0,1)$, thus $\mu$-a.e., $x^{a}$ tends to 0 as $a$ tends to $+\infty$ and is dominated by 1 everywhere.

3. If $a>b$, for all $x$ in $(0,1): x^{a}<x^{b}$ then this is true for $\mu$ a.e..

Proof of Lemma 2.3. This is a consequence of Lebesgue's dominated convergence theorem for holomorphic functions. For almost every real number $x>0: s \mapsto f(x) x^{s-1}$ is holomorphic in $\mathbb{C}$. Let $\eta>0$. For all $s$ in $\{s \in \mathbb{C} \mid a+1+\eta \leqslant \operatorname{Re} s \leqslant b+1-\eta\}$ and for almost every $x>0$ : $\left|f(x) x^{s-1}\right| \leqslant|f(x)| x^{a+\eta} \mathbf{1}_{x \in(0,1]}+|f(x)| x^{b-\eta} \mathbf{1}_{x \in[1,+\infty)} \in \mathbb{L}^{1}$. (In particular: $\left.\forall n \in \mathbb{N}^{*} \forall s \in \mathbb{C} \mid a+1<\operatorname{Re} s<b+1 \quad \mathcal{M} f^{(n)}(s)=\int_{0}^{+\infty}(\ln x)^{n} x^{s-1} f(x) \mathrm{d} x.\right)$

Proof of Lemma 2.4. Let $0<\eta<\frac{b-a}{2}$. For $\varphi=\mathbf{1}_{[c, d]}$, with $0<c \leqslant d, \mathcal{M} \varphi$ is holomorphic in $\mathbb{C}$ using Lemma 2.3 and can be explicitly computed $\left(\mathcal{M} \varphi(s)=\frac{1}{s}\left(c^{s}-d^{s}\right)\right.$ for $s \neq 0$ and $\left.\mathcal{M} \varphi(0)=\ln \left(\frac{d}{c}\right)\right)$; an easy computation leads to the desired property. By linearity of $\mathcal{M}$ it is also true for a linear combination of indicator functions.

Let $\varepsilon>0$. There exists a linear combination of indicator functions $\varphi$ such that $\|f-\varphi\|_{\mathbb{L}^{1}\left((0,1), x^{a+\eta} \mathrm{d} x\right)} \leqslant \varepsilon$ and $\|f-\varphi\|_{\mathbb{L}^{1}\left((1,+\infty), x^{b-\eta} \mathrm{d} x\right)} \leqslant \varepsilon$. Therefore

$$
|\mathcal{M}(f)(\zeta+i t)| \leqslant \int_{0}^{1}|(f-\varphi)(x)| x^{a+\eta} \mathrm{d} x+\int_{1}^{+\infty}|(f-\varphi)(x)| x^{b-\eta} \mathrm{d} x+|\mathcal{M}(\varphi)(\zeta+i t)|
$$

and then $\varlimsup_{|t| \rightarrow+\infty}|\mathcal{M}(f)(\zeta+i t)| \leqslant 2 \varepsilon$ uniformly with respect to $\zeta$ in $[a+1+\eta, b+1-$ $\eta]$.

\section{Proofs of subsections 3.1 and 3.2}

Proof of Proposition 3. (uniqueness) Let $q^{*}=q+1$ and $\mathcal{Z}=1-k \mathcal{M} \kappa_{0}$. The difference $w \in \mathbb{L}_{q}^{1}$ of two solutions of the equation $\mathcal{L} u=f$ satisfies $\mathcal{L} w=0$ so $\mathcal{Z}(s) \mathcal{M} w(s)=0$ for all $s$ in $q^{*}+i \mathbb{R}$. As $\mathcal{Z}$ does not vanish on $q^{*}+i \mathbb{R}$, it implies that $\mathcal{M} w=0$ on $q^{*}+i \mathbb{R}$, thus $w(x)=0$ for almost every $x>0$ by the $\mathbb{L}^{1}$ inversion theorem, $c f$. Theorem 2.2 Section 2 .

(explicit formula) Let $g(s)=\frac{\mathcal{M} f(s)}{\mathcal{Z}(s)}$. Since $\mathcal{M} f \in \mathbb{L}^{1}\left(q^{*}+i \mathbb{R}\right)$, and since thanks to Assumption $1, \mathcal{Z}^{-1}$ is bounded (by $1 / \delta$ ) on $q^{*}+i \mathbb{R}, g$ is also in $\mathbb{L}^{1}\left(q^{*}+i \mathbb{R}\right)$. We can thus define a function $u$ by the explicit formula (17).

(existence) Thanks to Assumption 2 there exists a function $v$ in $\mathbb{L}_{q}^{1}$ such that $\mathcal{M} f(s)(\mathcal{Z}(s))^{-1}=$ $\mathcal{M} v(s)$ on $q^{*}+i \mathbb{R}$ and then $u=v$ a.e., by the $\mathbb{L}^{1}$ inversion theorem; thus the previous explicit formula (17) defines an $\mathbb{L}_{q}^{1}$ function. 
Proof of Proposition 4. a) Let $g(s)=\frac{\mathcal{M} f(s)}{\mathcal{Z}(s)}$, which is meromorphic in $\{s \in \mathbb{C} \mid a<$ Re $s<b$ \} using Proposition 2.3, and may have poles in $P$. It results from Proposition 3 that Equation (11) $\mathcal{L}(u)=f$ has a unique solution $u_{q} \in \mathbb{L}_{q}^{1}$ for $q$ in $(a-1, c-1) \cup$ $(1, b-1)$ and that it is given for almost every $x>0$ by $u_{q}(x)=\frac{1}{2 \pi i} \int_{q^{*}-i \infty}^{q^{*}+i \infty} g(s) x^{-s} \mathrm{~d} s$.

b) Let $q_{1}, q_{2} \in(1, b-1)$. Denoting $q_{i}^{*}=q_{i}+1$, we want to show that, for almost every $x>0$ :

$$
\int_{q_{1}^{*}-i \infty}^{q_{1}^{*}+i \infty} g(s) x^{-s} \mathrm{~d} s=\int_{q_{2}^{*}-i \infty}^{q_{2}^{*}+i \infty} g(s) x^{-s} \mathrm{~d} s .
$$

For $n$ in $\mathbb{N}$ consider a rectangle $\Gamma_{n}$ oriented in the direct sense with edges at $q_{1}^{*} \pm$ $i c_{n}, q_{2}^{*} \pm i c_{n}$. As the function $\mathcal{Z}$ does not vanish in the strip $\left\{s \in \mathbb{C} \mid q_{1}^{*} \leqslant \operatorname{Re} s \leqslant q_{2}^{*}\right\}$ we have:

$0=\int_{\Gamma_{n}} g(s) x^{-s} \mathrm{~d} s=\int_{q_{1}^{*}-i c_{n}}^{q_{1}^{*}+i c_{n}} \cdots \mathrm{d} s-\int_{q_{2}^{*}-i c_{n}}^{q_{2}^{*}+i c_{n}} \cdots \mathrm{d} s+\int_{q_{1}^{*}-i c_{n}}^{q_{2}^{*}-i c_{n}} \cdots \mathrm{d} s-\int_{q_{1}^{*}+i c_{n}}^{q_{2}^{*}+i c_{n}} \cdots \mathrm{d} s$.

By Lemma 2.4, as $n$ tends to $+\infty$ the two horizontal integrals $\int_{q_{1}^{*}+i c_{n}}^{q^{*}+i c_{n}} g(s) x^{-s} \mathrm{~d} s$ and $\int_{q_{1}^{*}-i c_{n}}^{q_{*}^{*}-i c_{n}} g(s) x^{-s} \mathrm{~d} s$ tend to zero (if $\left|\mathcal{M} f\left(q^{*} \pm i t\right)\right| \leqslant \varepsilon$ for $|t| \geqslant c_{n}, q^{*} \in\left[q_{1}^{*}, q_{2}^{*}\right]$, these integrals are smaller than $\frac{1}{\delta}\left|q_{2}-q_{1}\right||x|^{-q_{1}^{*},-q_{2}^{*}} \varepsilon$ where $\left.x^{a, b}:=x^{a} \mathbf{1}_{x<1}+x^{b} \mathbf{1}_{x \geqslant 1}\right)$. By the dominated convergence theorem the two vertical integrals tend to the aimed integrals because $\int_{q_{1}^{*}-i \infty}^{q_{1}^{*}+i \infty}|\mathcal{M} f(s)| \mathrm{d} s$ and $\int_{q_{2}^{*}-i \infty}^{q_{2}^{*}+i \infty}|\mathcal{M} f(s)| \mathrm{d} s$ are finite. The same proof holds to show that $u_{q}$ does not depend on $q$ in $(a-1, c-1)$.

c) To show that $u_{l}-u_{r}$ is given by the above formula we use a path $\Gamma_{n}$ encircling the set $P$ : set $q_{1} \in(a-1, c-1)$ and $q_{2} \in(1, b-1)$ and consider a rectangle oriented in the direct sense with edges at $q_{1}^{*} \pm i c_{n}, q_{2}^{*} \pm i c_{n}$. The residue formula gives:

$$
\sum_{p \in P} \operatorname{Res}\left(g(s) x^{-s}, s=p\right)=\int_{q_{1}^{*}-i c_{n}}^{q_{1}^{*}+i c_{n}} \cdots-\int_{q_{2}^{*}-i c_{n}}^{q_{2}^{*}+i c_{n}} \cdots+\int_{q_{1}^{*}-i c_{n}}^{q_{2}^{*}-i c_{n}} \cdots-\int_{q_{1}^{*}+i c_{n}}^{q_{2}^{*}+i c_{n}} \cdots \mathrm{d} s .
$$

The horizontal integrals tend to 0 as $n$ goes to $+\infty$ because of Lemma 2.4 and because $t \mapsto 1-k \mathcal{M} \kappa_{0}\left(t+i c_{n}\right)$ is uniformly bounded from below for $t$ in $\left[q_{1}^{*}, q_{2}^{*}\right]$ and $n$ in $\mathbb{N}$ by Assumption 3. The vertical integrals converge for the same reason as above.

Proof of Proposition 5. (uniqueness) Let $\widetilde{q}=\frac{q+1}{2}$. The difference $w \in \mathbb{L}_{q}^{2}$ of two solutions of Equation (11) satifies $\mathcal{L}(w)=0$ so $\mathcal{Z}(s) \mathcal{M} w(s)=0$ for all $s$ in $\widetilde{q}+i \mathbb{R}$. As $\mathcal{Z}$ does not vanish in $\widetilde{q}+i \mathbb{R}$, it implies that $\mathcal{M} w=0$ in $\widetilde{q}+i \mathbb{R}$, thus $w(x)=0$ for almost every $x>0$ by Theorem 2.2 of Section 2 .

(existence) We define: $\mathcal{M} u(s)=\mathcal{M} f(s) \mathcal{Z}(s)^{-1}$ for all $s$ in $\widetilde{q}+i \mathbb{R}$. As $\mathcal{Z}^{-1}$ is bounded (by $1 / \delta)$ on $\widetilde{q}+i \mathbb{R}, \mathcal{M} u$ is in $\mathbb{L}^{2}(\widetilde{q}+i \mathbb{R})$ and thus, using Theorem 2.2 of Section 2 , $u$ is in $\mathbb{L}_{q}^{2}$. Moreover the function $u$ satisfies $\mathcal{M} f(s)=\mathcal{M} u(s) \mathcal{Z}(s)$ and is the solution of Equation (11).

(estimate) Using Theorem 2.2 of Section 2 we get the estimate:

$$
\begin{aligned}
\|f\|_{\mathbb{L}_{q}^{2}}^{2}=\frac{1}{2 \pi}\left\|\frac{\mathcal{M} u(s)}{\mathcal{Z}(s)}\right\|_{\mathbb{L}^{2}(\widetilde{q}+i \mathbb{R})}^{2} & \leqslant\left\|\mathcal{Z}(s)^{-2}\right\|_{\mathbb{L}^{\infty}(\widetilde{q}+i \mathbb{R})} \frac{1}{2 \pi}\|\mathcal{M} u(s)\|_{\mathbb{L}^{2}(\widetilde{q}+i \mathbb{R})}^{2} \\
& \leqslant\|\mathcal{Z}\|_{\mathbb{L}^{\infty}(\widetilde{q}+i \mathbb{R})}^{-2}\|u\|_{\mathbb{L}_{q}^{2}}^{2} .
\end{aligned}
$$


Proof of Proposition 6. Let $q \in(2 a-1,2 b-1)$ and $\widetilde{q}=\frac{q+1}{2} \in(a, b)$. Proposition 5 implies that Equation (11) with $f \in \mathbb{L}_{q}^{2}$ admits a unique solution $u_{q} \in \mathbb{L}_{q}^{2}$ for $q$ in $(2 a-1,2 c-1) \cup(3,2 b-1)$ and that it is defined by $u_{q}=\mathcal{M}^{-1}\left(\frac{\mathcal{M} u_{q}}{\mathcal{Z}}\right)$, with $\mathcal{Z}=1-k \mathcal{M} \kappa_{0}$, and where the inverse is taken on the line $\widetilde{q}+i \mathbb{R}$.

Let $T>0$. We define:

$$
f_{T}=f \mathbf{1}_{\left[\frac{1}{T}, T\right]} \text { on }[0,+\infty), \quad \varphi_{T}(s)=\exp \left(\frac{s^{2}}{T}\right), \quad g_{T}(s)=\frac{\mathcal{M} f_{T}(s)}{\mathcal{Z}(s)} \varphi_{T}(s) \text { on } \mathbb{C} .
$$

The function $f_{T}$ is in $\mathbb{L}_{q}^{1}$ for every $q$ in $\mathbb{R}$ thanks to Hölder's inequality. As a consequence the function $\mathcal{M} f_{T}$ is holomorphic on $\mathbb{C}$, is in $\mathbb{L}^{\infty}(q+i \mathbb{R})$ for every $q \in \mathbb{R}$ and the function $\mathcal{M} f_{T} \varphi_{T}$ is in $\mathbb{L}^{1}(q+i \mathbb{R})$ for every $q \in \mathbb{R}$ because

$$
\left|\varphi_{T}(q+i y)\right| \leqslant \exp \left(\frac{q^{2}-y^{2}}{T}\right) \in \mathbb{L}^{\infty}
$$

the function $g_{T}$ is meromorphic in $\left\{s \in \mathbb{C} \mid a^{*}<\operatorname{Re} s<b^{*}\right\}$ and may have poles in $P$.

Let $q_{1}$ and $q_{2}$ be in $(3,2 b-1)$. We want to show $u_{q_{1}}(x)=u_{q_{2}}(x)$ for almost every $x>0$. As in point $\mathrm{b}$ ) of the proof of Proposition 4 , taking paths along the lines $\widetilde{q}_{1}+i \mathbb{R}$ and $\widetilde{q}_{2}+i \mathbb{R}$, as $\mathcal{Z}$ is bounded from below on them, and as $g_{T} \in \mathbb{L}^{1}(\widetilde{q}+i \mathbb{R})$ for $q=q_{1}, q_{2}$, we get (using Lemma 2.4 but not Assumption 2):

$$
\mathcal{M}_{\widetilde{q}_{1}}^{-1}\left(g_{T}\right)=\mathcal{M}_{\widetilde{q}_{2}}^{-1}\left(g_{T}\right)
$$

Using the fact that the two definitions of the Mellin inverse transforms coincide for functions in $\mathbb{L}^{1} \cap \mathbb{L}^{2}$, we now pass to the limit when $T$ tends to $+\infty$. The function $\varphi_{T}(s)$ tends to 1 on every vertical strip $1+i \mathbb{R}$ as $T$ goes to $+\infty$. As $f$ is in $\mathbb{L}_{q}^{2}$ for every $q \in(2 a-1,2 b-1), f_{T}$ tends to $f$ in $\mathbb{L}_{q}^{2}$ and $\mathcal{M} f_{T}$ tends to $\mathcal{M} f$ in $\mathbb{L}^{2}(q+i \mathbb{R})$ for every $q \in(a, b)$.

As $\mathcal{Z}^{-1}$ is bounded on $\widetilde{q}_{1}+i \mathbb{R}$, the dominated convergence theorem shows that $\left(g_{T}\right)$ tends to $\frac{\mathcal{M} f}{\mathcal{Z}}$ in $\mathbb{L}^{2}\left(\widetilde{q}_{1}+i \mathbb{R}\right)$; then the sequence $\left(\mathcal{M}_{\widetilde{q}_{1}}^{-1}\left(g_{T}\right)\right)_{T}$ tends to $u_{q_{1}}$ in $\mathbb{L}_{q_{1}}^{2}$. We obtain similarly that $\left(\mathcal{M}_{\widetilde{q}_{2}}^{-1}\left(g_{T}\right)\right)_{T}$ tends to $u_{q_{2}}$ in $\mathbb{L}_{q_{2}}^{2}$. Up to extractions of subsequences we deduce that: $u_{q_{1}}=u_{q_{2}}$ almost everywhere. 1).

The same demonstration holds to show that $u_{q}$ does not depend on $q$ in $(a, 2(c+1)-$

\section{Study of the zeros of $1-k \mathcal{M}\left(\rho_{1} \delta_{\sigma_{1}}+\rho_{2} \delta_{\sigma_{2}}\right)$ along vertical lines}

Consider $\kappa_{0}=\rho_{1} \delta_{\sigma_{1}}+\rho_{2} \delta_{\sigma_{2}}$ with $0<\sigma_{1}<\sigma_{2}<1$ and $\sigma_{1} \leqslant \frac{1}{k} \leqslant \sigma_{2}$. The Mellin transform of $\kappa_{0}$ is $\mathcal{M} \kappa_{0}(s)=\rho_{1} \sigma_{1}^{s-1}+\rho_{2} \sigma_{2}^{s-1}$ for $s$ in $\mathbb{C}$. The assumptions $\mathcal{M} \kappa_{0}(1)=\rho_{1}+\rho_{2}=1$ and $\mathcal{M} \kappa_{0}(2)=\rho_{1} \sigma_{1}+\rho_{2} \sigma_{2}=\frac{1}{k}$ are satisfied iff $\left(\rho_{1}, \rho_{2}\right)=\left(\frac{\sigma_{2}-\frac{1}{k}}{\sigma_{2}-\sigma_{1}}, \frac{\frac{1}{k}-\sigma_{1}}{\sigma_{2}-\sigma_{1}}\right)$. The measure $\kappa_{0}$ is non-negative because $\sigma_{1} \leqslant \frac{1}{k} \leqslant \sigma_{2}$.

For a given real number $x$, we want to describe the sets $\Gamma_{x}=\left\{x+i y \mid y \in \mathbb{R}, \rho_{1} \sigma_{1}^{x} \sigma_{1}^{i y}+\right.$ $\left.\rho_{2} \sigma_{2}^{x} \sigma_{2}^{i y}=\frac{1}{k}\right\}$, which are the zeros of $1-k \mathcal{M}\left(\rho_{1} \delta_{\sigma_{1}}+\rho_{2} \delta_{\sigma_{2}}\right)$ on the line $x+i \mathbb{R}$. Each set $\Gamma_{x}$ is discrete because $\mathcal{M} \kappa_{0}$ is a non-constant holomorphic function. $\operatorname{As} \operatorname{supp}\left(\kappa_{0}\right)$ is 


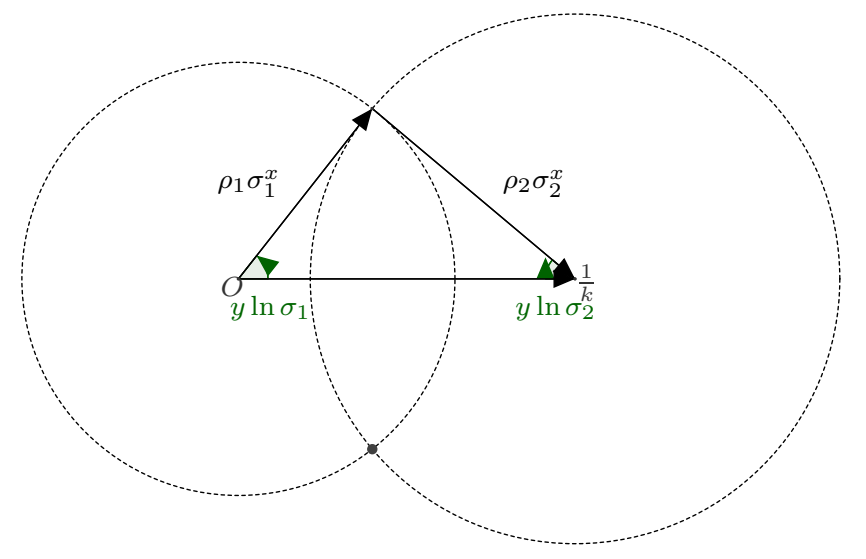

Figure 9: The triangle condition.

not included in $\{0,1\}$, the function $t \in \mathbb{R} \mapsto 1-k \mathcal{M} \kappa_{0}(t) \in \mathbb{R}$ is strictly increasing, $c f$. Lemma 2.2, so: $1 \in \Gamma_{1}$ (because $\mathcal{M} \kappa_{0}(1)=\frac{1}{k}$ ), $\Gamma_{x}$ is the empty set for $x>1$ and for every $x<1, \Gamma_{x} \cap \mathbb{R}$ is the empty set. Describing the set $\Gamma_{x}$ is based on the following geometrical fact.

Let $A, B$ and $C$ be non-negative real numbers. Finding a pair of real numbers $\left(\theta, \theta^{\prime}\right)$ satisfying $A e^{i \theta}+B e^{i \theta^{\prime}}-C=0$ is equivalent to building a positively oriented triangle, possibly flat, with sides of lengths $A, B, C$ and such that the oriented angles between the sides of lengths $C$ and $A$ and the sides of lengths $C$ and $B$ are respectively equal to $\theta$ and $\theta^{\prime}$. If $\Theta_{0}=\left(\theta_{0}, \theta_{0}^{\prime}\right)$ is a solution of the previous equation then all the solutions are $\Theta_{0}+2 \pi \mathbb{Z} \times 2 \pi \mathbb{Z} \cup-\Theta_{0}+2 \pi \mathbb{Z} \times 2 \pi \mathbb{Z}$ (in the complex plane the problem is to find the intersection of the circle centered at 0 of radius $A$ with the circle centered at $C$ of radius $B)$.

Let $x \in \mathbb{R}$ and set $A=\rho_{1} \sigma_{1}^{x}, B=\rho_{2} \sigma_{2}^{x}, C=\frac{1}{k}$. The equation $A \sigma_{1}^{i y}+B \sigma_{2}^{i y}=C$ has a solution iff one can find $y \in \mathbb{R}$ such that a triangle as above can be constructed with $\theta \equiv y \ln \sigma_{1} \bmod 2 \pi$ and $\theta^{\prime} \equiv y \ln \sigma_{2} \bmod 2 \pi($ triangle condition). If there exists a solution $y=y_{0}$ then $y$ is another solution iff $\left(y \ln \sigma_{1}, y \ln \sigma_{1}\right)-\left(y_{0} \ln \sigma_{1}, y_{0} \ln \sigma_{1}\right) \in(2 \pi \mathbb{Z})^{2}$ iff $\left(y-y_{0}\right)\left(\ln \sigma_{1}, \ln \sigma_{2}\right) \in(2 \pi \mathbb{Z})^{2}$ iff $y \in y_{0}+2 \pi G$ where $G$ is the additive subgroup of $\mathbb{R}$, $G=\alpha \mathbb{Z} \cap \beta \mathbb{Z}$ with $\alpha=\frac{1}{\ln \sigma_{1}}$ and $\beta=\frac{1}{\ln \sigma_{2}}$. The structure of such a group is discussed below. If there exists $y_{0}$ such that the triangle condition is true then $\Gamma_{x}=x+i H$ with $H=y_{0}+2 \pi G \cup-y_{0}+2 \pi G$. Otherwise $\Gamma_{x}=\emptyset$.

By the triangle condition, in order to have a solution we must have $|A-B| \leqslant \frac{1}{k} \leqslant$ $A+B$, where $A=\rho_{1} \sigma_{1}^{x}, B=\rho_{2} \sigma_{2}^{x}$. The right-hand side of this inequality is not satisfied for $x>1$, and we find again that $\Gamma_{x}$ is the empty set in this case. In the case $x=1$ the triangle condition is satisfied with $y=0$ so $\Gamma_{1}=1+2 \pi i G$ where $G$ is the same as above. In the case $x<1$, the triangle condition may be satisfied or not (for instance if $\left.|A-B|>\frac{1}{k}\right)$.

To finish describing the sets $\Gamma_{x}$ we have to study the subgroup of $\mathbb{R}, G=\alpha \mathbb{Z} \cap \beta \mathbb{Z}$ where $\alpha$ and $\beta$ are two given real numbers. The subgroups of $\mathbb{R}$ are either cyclic, that is of the form $c \mathbb{Z}$ for some real number $c \geqslant 0$, or are dense in $\mathbb{R}$. Therefore, as $G$ is discrete, 
it is cyclic. It is easy to prove that there exists $c>0$ such that $\alpha \mathbb{Z} \cap \beta \mathbb{Z}=c \mathbb{Z}$ iff $\alpha \neq 0$ and $\beta \neq 0$ and $\frac{\alpha}{\beta} \in \mathbb{Q}$.

We then have two cases. The first one is when $\frac{\ln \sigma_{2}}{\ln \sigma_{1}}$ is a rational number (for instance if $\sigma_{2}=1-\sigma_{1}$ and $\sigma_{1}=\frac{1}{\varphi}=\frac{\sqrt{5}-1}{2}, r-\frac{1}{3 r}, r^{\prime}-\frac{5}{9 r^{\prime}}+\frac{1}{3}$ where $\left.r=\sqrt[3]{\frac{1}{2}+\frac{\sqrt{93}}{18}}, r^{\prime}=\sqrt[3]{\frac{11}{54}+\frac{\sqrt{69}}{18}}\right) ;$ then $G=\alpha \mathbb{Z} \cap \beta \mathbb{Z}$ is cyclic and not reduced to $\{0\}$. The set $\Gamma_{x}$ is $x+i H$ or is the emptyset whether the triangle condition is satisfied or not. The second (and generic) case is when $\frac{\ln \sigma_{2}}{\ln \sigma_{1}}$ is not a rational number (for instance if $\sigma_{2}=1-\sigma_{1}$ with $\sigma_{1}$ transcendental); then $G$ is reduced to $\{0\}$ and consequently $\Gamma_{1}=\{1\}$ and for $x<1, \Gamma_{x}=x \pm i y_{0}$ or $\Gamma_{x}=\emptyset$ whether the triangle solution is satisfied or not.

\section{E Inverting the operator $\mathcal{L}$ as a Neumann series}

It is possible to build directly $\mathcal{L}^{-1}: \mathbb{L}_{q}^{p} \rightarrow \mathbb{L}_{q}^{p}$ for any $p \geqslant 1$ and for large enough weights $q$ $(q>2 p-1)$ thanks to a Neumann series. This result generalises Proposition 2.1. of [15], which corresponds to the case $p=2$ and $q>3$, and was proved by the use of Young's inequalities and the Lax-Milgram theorem.

The following constants in $\mathbb{R} \cup\{ \pm \infty\}$ are defined in the article [15].

Definition E.1. Let $\kappa$ be a general fragmentation kernel. We set, for a real number $u$ :

$$
C(u)=\sup _{x>0} \int_{x}^{+\infty} \kappa(x, y)\left(\frac{x}{y}\right)^{u} \mathrm{~d} y, \quad D(u)=\sup _{y>0} \int_{0}^{y} \kappa(x, y)\left(\frac{x}{y}\right)^{u} \mathrm{~d} x .
$$

Remark 10. In the case of a self-similar kernel $\kappa(x, y)=\frac{1}{y} \kappa_{0}\left(\frac{x}{y}\right)$ the constants of definition E.1 are simply written:

$$
C(u)=\mathcal{M} \kappa_{0}(u), \quad D(u)=\mathcal{M} \kappa_{0}(u+1) .
$$

Proposition 7. Let $p, r$ be two real numbers with $1<p<\infty$. The linear operator $\mathcal{K}: \mathbb{L}_{q}^{p} \rightarrow \mathbb{L}_{q}^{p}$ defined by (7) has a norm smaller than

$$
C_{r}:=C\left(\frac{q+1}{p}-r\right)^{1 / p^{\prime}} D\left(\frac{q+1}{p}+r(p-1)-1\right)^{1 / p}
$$

where $C(u), D(u)$ are introduced in definition E.1 and where $p^{\prime}$ is the conjugate exponent of $p$ (that is: $\frac{1}{p^{\prime}}+\frac{1}{p}=1$ ).

If there exists a real number $r$ such that $C_{r}<\frac{1}{k}$, the linear operator $\mathcal{L}: \mathbb{L}_{q}^{p} \rightarrow \mathbb{L}_{q}^{p}$ defined by (8) is invertible and we have the estimate:

$$
\left\|\mathcal{L}^{-1}\right\|_{\mathbb{L}_{q}^{p} \rightarrow \mathbb{L}_{q}^{p}} \leqslant\left(1-k C_{r}\right)^{-1}
$$

Proof. Let $u \in \mathbb{R}$. Writing $\kappa=\kappa^{1 / p^{\prime}}\left(\frac{x}{y}\right)^{u / p^{\prime}} \kappa^{1 / p}\left(\frac{x}{y}\right)^{-u / p^{\prime}}$ for $x, y>0$ and using Hölder's inequality we have:

$$
\begin{gathered}
\int_{x}^{+\infty} \kappa(x, y)|H(y)| \mathrm{d} y \leqslant \\
\left(\int_{x}^{+\infty} \kappa(x, y)\left(\frac{x}{y}\right)^{u} \mathrm{~d} y\right)^{1 / p^{\prime}}\left(\int_{x}^{+\infty} \kappa(x, y)\left(\frac{x}{y}\right)^{-u p / p^{\prime}}|H(y)|^{p} \mathrm{~d} y\right)^{1 / p} .
\end{gathered}
$$


Then using Fubini's theorem for non-negative functions:

$$
\begin{aligned}
\|\mathcal{K} H\|_{\mathbb{L}_{q}^{p}}^{p} & \leqslant C(u)^{\frac{p}{p^{\prime}}} \int_{0}^{+\infty} x^{q} \int_{x}^{+\infty} \kappa(x, y)\left(\frac{x}{y}\right)^{-u(p-1)}|H(y)|^{p} \mathrm{~d} y \mathrm{~d} x \\
& =C(u)^{\frac{p}{p^{\prime}}} \int_{y>0}|H(y)|^{p} y^{q} \int_{0}^{y} \kappa(x, y)\left(\frac{x}{y}\right)^{-u(p-1)+q} \mathrm{~d} x \mathrm{~d} y \\
& \leqslant C(u)^{\frac{p}{p^{\prime}}} D(-u(p-1)+q)\|H\|_{\mathbb{L}_{q}^{p}}^{p}
\end{aligned}
$$

This calculation leads to the conclusion by taking $r=\frac{q+1}{p}-u$.

In the Banach space $\mathcal{L}\left(\mathbb{L}_{q}^{p}, \mathbb{L}_{q}^{p}\right)$ with the operator norm \|\| , as $\|k \mathcal{K}\| \leqslant k C_{r}<1$, we can write the Neumann series $\mathcal{L}^{-1}=(\mathrm{Id}-k \mathcal{K})^{-1}=\sum_{p=0}^{+\infty} k^{p} \mathcal{K}^{p}$ which gives the second point of the proposition taking \|\| .

Remark 11. If we have $f$ in $\mathbb{L}_{q}^{p}$ we can define the affine map $T: \mathbb{L}_{q}^{p} \rightarrow \mathbb{L}_{q}^{p}$ by $T(u)=$ $f+k \mathcal{K}(u)$. By the previous calculations $T$ is Lipschitz continuous with its constant less than $k C_{r}$ and is therefore a contraction if this constant is strictly less than one. Let us call $u$ the unique fixed point of $T$, which is the solution of $\mathcal{L} u=f$. Defining the sequence $u_{l+1}=T\left(u_{l}\right)$ for some $u_{0}$ in $\mathbb{L}_{q}^{p}$, we have that $\left(u_{l}\right)$ tends to $u$ with geometric convergence rate: $\left\|u_{l}-u\right\|_{\mathbb{L}_{q}^{p}} \leqslant \frac{\left(k C_{r}\right)^{l}}{1-k C_{r}}\left\|u_{1}-u_{0}\right\|_{\mathbb{L}_{q}^{p}}$.

This remark was used to implement a code to compute $\mathcal{L}^{-1} f$ for a given $f$ in $\mathbb{L}_{q}^{p}$. The inverse obtained in this way is in $\mathbb{L}_{q}^{p}$ for large enough values of $q$.

Corollary 1. Let $p$ be a real number with: $1<p<\infty$ and let $\kappa(x, y)=\frac{1}{y} \kappa_{0}\left(\frac{x}{y}\right)$ be a selfsimilar kernel defined by (5). The linear operator $\mathcal{L}: \mathbb{L}_{q}^{p} \rightarrow \mathbb{L}_{q}^{p}$ defined by (8) is invertible if $\mathcal{M} \kappa_{0}(\widehat{q})<\frac{1}{k}$ where $\widehat{q}=\frac{q+1}{p}$ with the estimate: $\left\|\mathcal{L}^{-1}\right\|_{\mathbb{L}_{q}^{p} \rightarrow \mathbb{L}_{q}^{p}} \leqslant\left(1-k \mathcal{M} \kappa_{0}(\widehat{q})\right)^{-1}$. Moreover if $\operatorname{supp}\left(\kappa_{0}\right)$ is not included in $\{0,1\}$ the condition $\mathcal{M} \kappa_{0}(\widehat{q})<\frac{1}{k}$ is satisfied iff $q>2 p-1$.

Proof. The first part results from Proposition 7 for $r=0$ and from Remark 10. The second part is a consequence of Lemma 2.2: $\mathcal{M} \kappa_{0}(\widehat{q})<\frac{1}{k}=\mathcal{M} \kappa_{0}(2)$ iff $\widehat{q}>2$.

Remark 12. For the conservative equation introduced in Remark 9 with a self-similar kernel $\kappa(x, y)=\frac{1}{y} \kappa_{0}\left(\frac{x}{y}\right)$ Corollary 1 becomes: the linear operator $\mathcal{L}=\mathrm{Id}-\mathcal{K}: \mathbb{L}_{q}^{p} \rightarrow \mathbb{L}_{q}^{p}$ defined by $(7)$ is invertible if $\mathcal{M} \kappa_{0}(\widehat{q})<1$ where $\widehat{q}=\frac{q+1}{p}$. If $\operatorname{supp}\left(\kappa_{0}\right)$ is not included in $\{0,1\}$ the condition $\mathcal{M} \kappa_{0}(\widehat{q})<1$ is satisfied iff $q>p-1$.

For a self-similar kernel $\kappa_{0}$ such that $\operatorname{supp}\left(\kappa_{0}\right)$ is not included in $\{0,1\}$ the condition of this corollary is true for any fixed $p$ in $(1,+\infty)$ and for large enough values of $q$, unlike the conditions obtained in Propositions 3 and 5 which are satisfied only for $p=1$ and 2 but for any weight $q$ except a countable set of values of $q$.

\section{References}

[1] F. Baccelli, D. R. McDonald, and J. Reynier. A mean-field model for multiple tcp connections through a buffer implementing red. Performance Evaluation, 49(1-4):77 $-97,2002$. 
[2] D. Balagué, J. Cañizo, and P. Gabriel. Fine asymptotics of profiles and relaxation to equilibrium for growth-fragmentation equations with variable drift rates. Kinetic Related Models, 2013. in press.

[3] H.T. Banks, K.L. Sutton, W.C. Thompson, G. Bocharov, D. Roosec, T. Schenkeld, and A. Meyerhanse. Estimation of cell proliferation dynamics using cfse data. Bull. of Math. Biol., 2010.

[4] George I. Bell and Ernest C. Anderson. Cell growth and division: I. a mathematical model with applications to cell volume distributions in mammalian suspension cultures. Biophysical Journal, 7(4):329 - 351, 1967.

[5] M. J. Cáceres, J. A. Cañizo, and S. Mischler. Rate of convergence to the remarkable state for fragmentation and growth-fragmentation equations. Journal de Mathematiques Pures et Appliquees, 96(4):334-362, 2011.

[6] V. Calvez, N. Lenuzza, D. Oelz, J.-P. Deslys, P. Laurent, F. Mouthon, and B. Perthame. Size distribution dependence of prion aggregates infectivity. Math. Biosci., 1:88-99, 2009.

[7] G. Chiorino, J. A. J. Metz, D. Tomasoni, and P. Ubezio. Desynchronization rate in cell populations: mathematical modeling and experimental data. J. Theor. Biol., 208:185-199, 2001.

[8] B. Cloez. Limit theorems for some branching measure-valued processes. arXiv:1106.0660v2, 2012.

[9] M. Crouzeix and A. L. Mignot. Analyse numérique des équations différentielles. Masson, Paris, 1984.

[10] M. Doumic and P. Gabriel. Eigenelements of a general aggregation-fragmentation model. Mathematical Models and Methods in Applied Sciences, 20(05):757, 2009.

[11] M. Doumic, M. Hoffmann, N. Krell, and L. Robert. Statistical estimation of a growth-fragmentation model observed on a genealogical tree. submitted, 2013.

[12] M. Doumic, M. Hoffmann, P. Reynaud, and V. Rivoirard. Nonparametric estimation of the division rate of a size-structured population. SIAM J. on Numer. Anal., 50(2):925-950, 2012.

[13] M. Doumic, P. Maia, and J.P. Zubelli. On the calibration of a size-structured population model from experimental data. Acta Biotheoretica, 58(4):405-413, 2010.

[14] M. Doumic, B. Perthame, and J.P. Zubelli. Numerical Solution of an Inverse Problem in Size-Structured Population Dynamics. Inverse Problems, 25(4):045008, 2009.

[15] M. Doumic and L.M. Tine. Estimating the division rate for the growth-fragmentation equation. Journal of Mathematical Biology, published online, 2012.

[16] H.W. Engl, M. Hanke, and A. Neubauer. Regularization of inverse problems, volume 375 of Mathematics and its Applications. Springer, 1996.

[17] A. Groh, J. Krebs, and M. Wagner. Efficient solution of an inverse problem in cell population dynamics. Inverse Problems, 27, 2011. 
[18] P. Laurençot and B. Perthame. Exponential decay for the growth-fragmentation/celldivision equation. Comm. Math. Sc., 7(2):503-510, 2009.

[19] J. A. J. Metz and O. Diekmann, editors. The dynamics of physiologically structured populations, volume 68 of Lecture Notes in Biomathematics. Springer-Verlag, Berlin, 1986. Papers from the colloquium held in Amsterdam, 1983.

[20] P. Michel, S. Mischler, and B. Perthame. General relative entropy inequality: an illustration on growth models. J. Math. Pures Appl. (9), 84(9):1235-1260, 2005.

[21] K. Pakdaman, B. Perthame, and D. Salort. Dynamics of a structured neuron population. Nonlinearity, 23(1):55-75, 2010.

[22] K. Pakdaman, B. Perthame, and D. Salort. Relaxation and self-sustained oscillations in the time elapsed neuron network model. (submitted), 2011.

[23] K. Pakdaman, B. Perthame, and D. Salort. Adaptation and fatigue model for neuron networks and large time asymptotics in a nonlinear fragmentation equation. (submitted), 2012.

[24] B. Perthame and L. Ryzhik. Exponential decay for the fragmentation or cell-division equation. J. Differential Equations, 210(1):155-177, 2005.

[25] B. Perthame and J.P. Zubelli. On the inverse problem for a size-structured population model. Inverse Problems, 23(3):1037-1052, 2007.

[26] L. Robert, M. Hoffmann, N. Krell, S. Aymerich, J. Robert, and M. Doumic. Division control in escherichia coli is based on a size-sensing rather than timing mechanism. submitted, 2013.

[27] W. Rudin. Fourier Analysis on Groups. Interscience Publishers, Inc., New York, 1962.

[28] Eric J Stewart, Richard Madden, Gregory Paul, and François Taddei. Aging and death in an organism that reproduces by morphologically symmetric division. PLoS Biology, 3(2):e45, 2005.

[29] E. C. Titchmarsch. Introduction to the theory of Fourier integrals. The Clarendon Press, 1937.

[30] C. Villani. Cours de deuxième année donné à l'Ecole normale supérieure de Lyon. http://cedricvillani.org/wp-content/uploads/2013/03/ana2.pdf, 2003. Accessed: 2013-05-14.

[31] G. Wahba. Practical approximate solutions to linear operator equations when the data are noisy. SIAM J. Numer. Anal., 14:651-667, 1977. 